\title{
Combination of Chemotherapy and Photodynamic Therapy with Oxygen Self-Supply in the Form of Mutual Assistance for Cancer Therapy
}

\author{
Ying Chen ${ }^{\mathrm{I}-3}$ \\ Lei Zhang $^{\text {I-3 }}$ \\ Fangxuan Li (iD) 4 \\ Jindong Sheng' \\ Changxiao $\mathrm{Xu}^{\prime}$ \\ Dan $\mathrm{Li}^{\prime}$ \\ $\mathrm{Hu}$ Yu' \\ Wenxin Liu' \\ 'Department of Gynecologic Oncology, \\ Tianjin Medical University Cancer \\ Institute and Hospital, Tianjin, 300060, \\ People's Republic of China; ${ }^{2}$ Tianjin's \\ Clinical Research Center for Cancer, Key \\ Laboratory of Cancer Prevention and \\ Therapy, Tianjin, 300060, ${ }^{3}$ National \\ Clinical Research Centre of Cancer, \\ Tianjin, 300060, People's Republic of \\ China; ${ }^{4}$ Department of Cancer \\ Prevention, Tianjin Medical University \\ Cancer Institute and Hospital, Tianjin, \\ 300060, People's Republic of China
}

Correspondence: Ying Chen Department of Gynecologic Oncology, Tianjin Medical University Cancer Institute and Hospital, Huanhuxi Road, Hexi District, Tianjin, 300060, People's Republic of China

Email lychenying2004@I26.com
Introduction: Photodynamic therapy (PDT) has been widely researched by cancer therapists in recent years. This study aims to establish a drug delivery system combining PDT and chemotherapy to show that chemotherapeutic drugs provide oxygen to PDT, while PDT promotes the release of chemotherapeutic drug.

Methods: Firstly, poly(ethylene glycol)-lysine(Ce6)-block-poly(L-glutamate)-imidazole (mPEG-lys(Ce6)-PGA-AIM) was synthesized and self-assembled into micelles that exhibited $\mathrm{pH}-$ and ROS-responsiveness and buffering capacity. Perfluorohexanoate-modified cisplatin (FCP), as oxygen carriers, was encapsulated into mPEG-lys(Ce6)-PGA-AIM micelles. Then, the properties of micelles and their biological functions in vivo and in vitro were investigated.

Results: The micelles exhibited remarkabe stability, $\mathrm{pH}$ regulated drug release, good biocompatibility and effective tumor penetration. Cellular uptake demonstrated the efficient endosome/lysosome escape of CFMs, which facilitates the intracellular drug release. Both in vitro and in vivo experiments reflected that CFMs with laser irradiation showed significantly improved therapeutic activity compared with single PDT or chemotherapy.

Conclusion: Chemotherapy and PDT were combined in the form of mutual assistance to provide a promising strategy for clinical treatment.

Keywords: chemotherapy, photodynamic therapy, dual-responsiveness, oxygen carriers

\section{Introduction}

Photodynamic therapy (PDT) has been widely researched by cancer therapists in recent years. Photosensitizers absorb energy from the laser and convert oxygen into cytotoxic reactive oxygen species (ROS) to kill cancer cells. ${ }^{1,2}$ The main advantages of PDT are the ability to control the exposure area and duration of light to maximize the therapeutic effect and reduce damage to normal tissues, especially compared with conventional strategies, such as chemotherapy. However, the effectiveness of PDT is largely limited by the amount of oxygen in solid tumors. ${ }^{3-5}$ The situation becomes worse after PDT consumes oxygen, which aggravates the hypoxia of the tumor and causes PDT to eventually lose its effect.

In view of tumor hypoxia, some strategies have been developed, such as hyperbaric oxygen inhalation or multiple step-by-step irradiations of light. ${ }^{6}$ Besides, another strategy has been developed to alleviate the hypoxic environment 
of tumors based on oxygen generating materials. As reported, catalase-integrated HA-CAT@aCe6 NPs have been successfully developed to reduce hypoxia to ensure high efficacy of PDT in vitro and in vivo. ${ }^{7}$ Intelligent $\mathrm{MnO}_{2}$ nanosheets anchored with upconversion nanoprobes have also been designed for the concurrent stimuliresponsive imaging/therapy of solid tumors by overcoming hypoxia. ${ }^{8}$ Besides, recent studies have shown that perfluorocarbons (PFCs), as oxygen tanks, are capable of enriching high levels of oxygen because of the weak molecular cohesion in PFC, thus facilitating the insertion of $\mathrm{O}_{2}$ molecules. ${ }^{9}$ However, PFCs usually need to be formulated into emulsions and exhibit rapid release of oxygen. ${ }^{10}$ Against these defects of PFCs, PEGylated fluorinated poly ( $\beta$-amino ester)s (PF-PEG) was synthesized, based on which a multifunctional nanoplatform with large oxygen-carrying capacity was established for enhanced PDT therapy. $^{11}$

In addition to relieving tumor hypoxia, numerous research work has been devoted to the combination of chemotherapy and PDT, and great progress has been made. ROS-sensitive thioketal-linked polyphosphoesterdoxorubicin (DOX) conjugate has been prepared for precisely photo-triggered locoregional chemotherapy. ${ }^{12}$ Under irradiation of light, thioketal linkage was cut off by generated ROS and the conjugated DOX was released to facilitate the chemotherapy. A cisplatin drug delivery system with near-infrared light-stimuli drug release property was developed by encapsulating cisplatin and Indocyanine Green (ICG) in the novel tellurium-containing polymer (PEG-PUTe-PEG) based nanoparticles. ${ }^{13}$ Tellurium is easily oxidized by ROS under the stimulation of a nearinfrared laser at the tumor site and thus cisplatin could be rapidly released from the nanocarriers with the oxidation. Furthermore, dual-responsive polymeric micelles have been prepared for enhanced combined delivery of doxorubicin and zinc (II) phthalocyanine in a tumor-bearing mice model. ${ }^{14}$ Upon laser irradiation and the reduction of glutathione (GSH), the hydrophobic segments of micelles became hydrophilic, leading to micelle disassembly and cargo release. In these studies, PDT was responsible for promoting the release of chemotherapeutic drugs in different ways to achieve better anti-tumor effects. However, what can chemotherapy do for PDT?

In our research, we aim to establish a drug delivery system combining PDT and chemotherapy to show that chemotherapeutic drugs provide oxygen to PDT, while PDT promotes the release of chemotherapeutic drugs.
Firstly, mPEG-lys(Ce6)-PGA-AIM was synthesized to contain imidazoles that provide cross-linking sites with $\mathrm{Zn}^{2+}$ and proton sponge effects which considered that nanocarriers containing imidazole can improve cellular uptake and rapid endosomal escape. ${ }^{15}$ More importantly, imidazoles, as the hydrophobic segments, could be oxidized by ROS, which promoted micelle disassembly and achieved fast drug release. ${ }^{16}$ Secondly, perfluorohexanoic acid modified cisplatin (FCP) was synthesized as the chemotherapeutic prodrug and oxygen generation materials. Finally, FCP was loaded into mPEG-lys(Ce6)-PGA-AIM micelles and $\mathrm{Zn}^{2+}$ was added to form core cross-linked FCP-loaded micelles (CFMs). As demonstrated in Figure 1, the cross-linkage stabilized micelles in the process of blood circulation and avoided the premature release of the drug. Negative surface charge of these micelles could reduce the protein adsorption and avoid capture by the reticuloendothelial system (RES). Due to the enhanced permeability and retention (EPR) effect, these micelles would accumulate in tumor tissue, where the negative surface charge would be converted to positive surface charge in the weakly acidic environment of tumor tissue. After CFMs were internalized into tumor cells, imidazoles began protonation, promoting the endosome/ lysosome escape. Meanwhile, conjugated Ce6 would generate ROS under laser irradiation with the aid of oxygen. These generated ROS further induced the imidazole-urea transformation and changed the hydrophobicity/hydrophilicity balance to achieve a rapid release of FCP. Finally, generated ROS and FCP jointly inhibit tumor growth.

\section{Materials and Methods \\ Materials}

Methoxy (polyethylene glycol) amine (mPEG-NH 2 , MW 5000), Fmoc-lys(Boc)-OH, 5-benzyl-L-glutamate (BLG) and 2-hydroxypyridine (2-HP) were bought from Shanghai McLean biochemical Technology Co., Ltd. N, N'Dicyclohexylcarbodiimide (DCC), $N$-hydroxysuccinimide (NHS) and trifluoroacetic acid (TFA) were purchased from Energy Chemical. 1-(3-Aminopropyl) imidazole (AIM), hexanoic anhydride (HA) and perfluorohexanoic anhydride (PHA) were bought from Mayer Chemical Technology (Shanghai, China). Chlorine e6 (Ce6) and cisplatin were obtained from Sigma-Aldrich. Diethylamine (DEA) and triphosgene were bought from Aladdin Biochemical Technology (Shanghai, China). All solvents, including dichloromethane (DCM), diethyl ether, triethylamine 


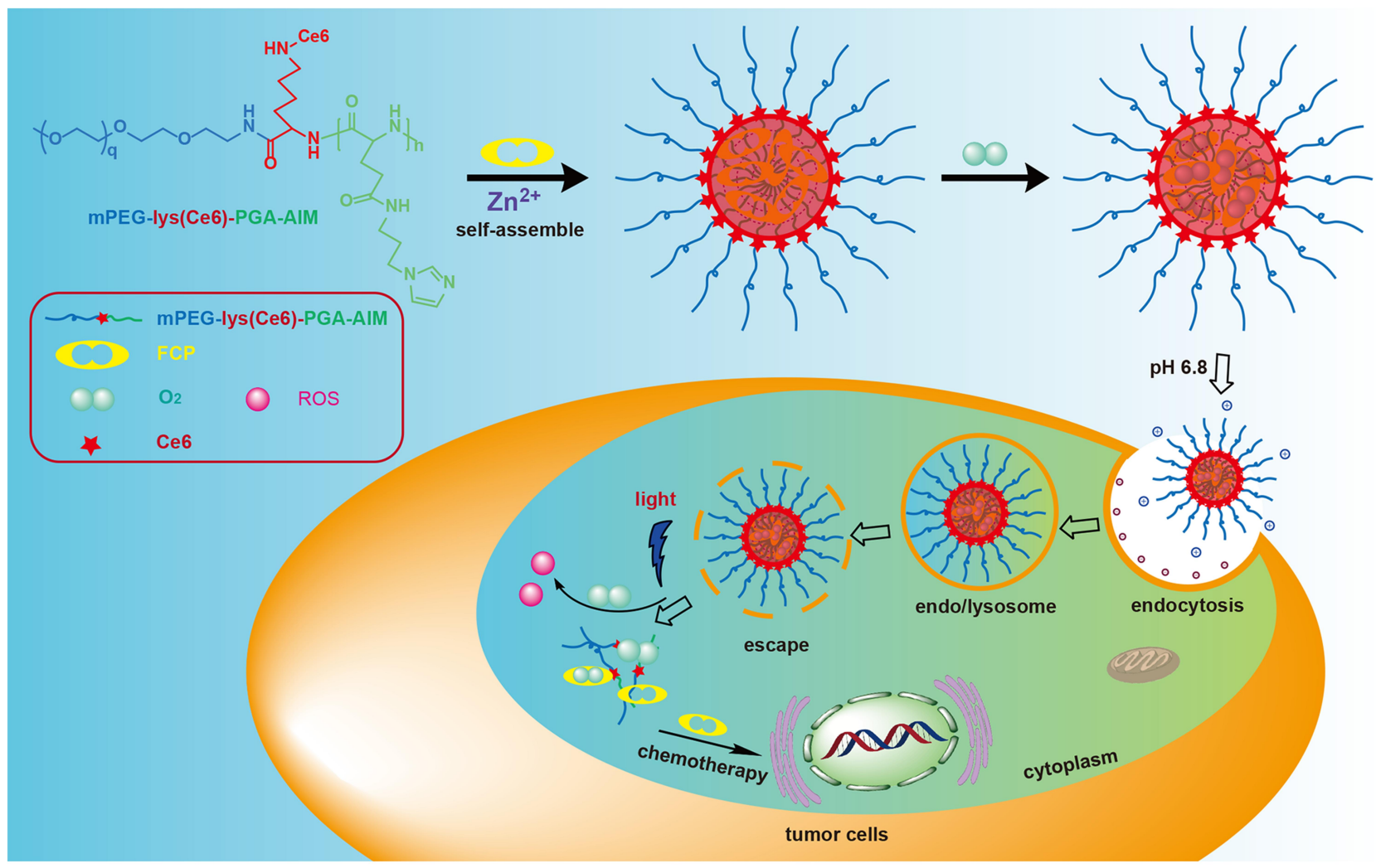

Figure I The chemical structure of mPEg-Lys (Ce6)-PGA-AIM and the mechanism of drug delivery system combining chemotherapy and PDT.

(TEA) and $N, N$-dimethylformamide (DMF) were bought from General-reagent. SKOV3 cells were purchased from Procell (Wuhan, China).

\section{Synthesis of mPEG-Lys (Boc/Fmoc)}

Before synthesis of mPEG-lys (Boc/Fmoc), Fmoc-Lys (Boc)-OH was treated by DCC/NHS method. In short, Fmoc-Lys (Boc)-OH (0.8 g, $1.7 \mathrm{mmol})$ and NHS $(0.3 \mathrm{~g}$, $2.6 \mathrm{mmol})$ were dissolved in $20 \mathrm{~mL}$ DCM. DCC $(0.8 \mathrm{~g}$, $3.9 \mathrm{mmol}$ ) was added and reacted for $12 \mathrm{~h}$. After filtration, the mixture was concentrated and mixed with $\mathrm{mPEG- \textrm {NH } _ { 2 }}$ $(2 \mathrm{~g}, 0.4 \mathrm{mmol})$ in $10 \mathrm{~mL}$ DCM. After stirring for another $24 \mathrm{~h}$, the reaction was filtrated and rinsed 2 times with a saturated $\mathrm{NaCl}$ solution. Organic phase was gathered and dried with anhydrous $\mathrm{Na}_{2} \mathrm{SO}_{4}$. mPEG-lys (Boc/Fmoc) was obtained by reprecipitation into excess diethyl ether. Yield: $92.1 \%$.

\section{Synthesis of mPEG-Lys (Boc)}

mPEG-lys (Boc/Fmoc) (1 g) was dissolved in $10 \mathrm{~mL}$ DCM followed by addition of $5 \mathrm{~mL}$ DEA. After stirring at room temperature for $4 \mathrm{~h}$, the reaction was washed with saturated $\mathrm{NaCl}$ solution twice. Then, organic phase was gathered and dehydrated with anhydrous $\mathrm{Na}_{2} \mathrm{SO}_{4}$. After concentration, the residue was added into excess diethyl ether three times to obtain mPEG-lys (Boc). Yield: 76.0\%.

\section{Synthesis of mPEG-Lys(Boc)-PBLG}

mPEG-lys(Boc)-PBLG was synthesized by two steps. Firstly, BLG-NCA was synthesized according to the report. ${ }^{17}$ Under $\mathrm{N}_{2}$ atmosphere, mPEG-lys (Boc) (0.85 g, $0.16 \mathrm{mmol})$ and BLG-NCA $(1.68 \mathrm{~g}, 6.4 \mathrm{mmol})$ were dissolved into $15 \mathrm{~mL}$ DMF at $35^{\circ} \mathrm{C}$ for $72 \mathrm{~h}$. After concentration, the residue was re-dissolved in DCM and reprecipitated into excess diethyl ether. mPEG-lys (Boc)PBLG was obtained by vacuum drying. Yield: $86.9 \%$.

\section{Synthesis of mPEG-Lys-PBLG}

The deprotection was performed according to the typical method. Briefly, mPEG-lys (Boc)-PBLG (1.2 g) was dissolved into $10 \mathrm{~mL}$ DCM and $5 \mathrm{~mL}$ TFA was added. The mixture was stirred at room temperature for $4 \mathrm{~h}$. After concentration under reduced pressure, the residue was redissolved into DCM and reprecipitated into excess diethyl ether. mPEG-lys-PBLG was obtained by vacuum drying. Yield: $93.2 \%$. 


\section{Synthesis of mPEG-Lys (Ce6)-PBLG}

Ce6 (0.06 g, $0.1 \mathrm{mmol})$ was mixed with DCC $(0.03 \mathrm{~g}, 0.15$ $\mathrm{mmol})$ in $5 \mathrm{~mL}$ DMSO. After adding NHS $(0.013 \mathrm{~g}, 0.11$ mmol), the reaction was mixed at room temperature for 4 h. After adding the PEG-lys-PBLG $(1.2 \mathrm{~g}, 0.09 \mathrm{mmol})$ in $10 \mathrm{~mL}$ DMSO with $12.5 \mu \mathrm{L}$ TEA, the reaction was mixed for $24 \mathrm{~h}$, then filtrated and dialyzed (MW 5000) against DMSO for $24 \mathrm{~h}$ with DMSO changed three times and against pure water for another $24 \mathrm{~h}$ with water changed four times . mPEG-lys (Ce6)-PBLG was obtained by lyophilization. Yield: $83.4 \%$.

\section{Synthesis of mPEG-Lys (Ce6)-PGA-AIM}

mPEG-lys (Ce6)-PBLG and AIM were dissolved in $5 \mathrm{~mL}$ anhydrous DMF plus 2-HP. Under $\mathrm{N}_{2}$ atmosphere, the reaction was mixed at $40{ }^{\circ} \mathrm{C}$ for $48 \mathrm{~h}$. After that, the reaction mixture was dialyzed in pure water for 48 $\mathrm{h}$ with water changed five times. mPEG-lys (Ce6)-PGAAIM was obtained by lyophilization. Yield: $71.6 \%$.

\section{Synthesis of HCP and FCP}

HCP and RCP were synthesized according to the report. ${ }^{18-20}$ Cisplatin $(0.69 \mathrm{~g}, 2.05 \mathrm{mmol})$ was firstly oxidized by $\mathrm{H}_{2} \mathrm{O}_{2}$. Then, the cis-platinum oxide solution in DMSO was mixed with $10 \mathrm{~mL}$ of the corresponding aliphatic anhydride in DMF: HA and PHA. After stirring for $48 \mathrm{~h}$ at room temperature, the mixture was mixed with $20 \mathrm{~mL}$ water to precipitate a pale-yellow solid, which was then filtered and separated. The yellow solid was washed several times with diethyl ether and dried. Yield: HCP $53.4 \%$; FCP $49.1 \%$.

\section{Preparation Micelles}

Blank micelles (BMs), HCP-loaded (HMs) micelles and FCP-loaded (FMs) micelles were made through the dialysis method. Briefly, $10 \mathrm{mg}$ of mPEG-lys(Ce6)-PGA-AIM was dissolved in $2 \mathrm{~mL}$ DMSO. After that, BMs were collected by lyophilization and stored at $4{ }^{\circ} \mathrm{C}$ for use. $10 \mathrm{mg}$ of mPEG-lys(Ce6)-PGA-AIM and $1 \mathrm{mg} \mathrm{HCP}$ or FCP were dissolved in $2 \mathrm{~mL}$ DMSO, followed by filtration through $0.45 \mu \mathrm{m}$ filter and dialysis against pure water. HMs and FMs were obtained by lyophilization and stored at $4{ }^{\circ} \mathrm{C}$ for use. Core cross-linked micelles were prepared according to the report. ${ }^{16}$ Solution of $\mathrm{Zn}^{2+}$ was added into BMs, HMs and FMs suspension, followed by dialysis to remove redundant $\mathrm{Zn}^{2+}$ to generate $\mathrm{CMs}$, CHMs and CFMs, respectively.
Reverse-phase high-performance liquid chromatography (HPLC) was used to detect the drug loading content (DLC) and drug loading efficiency (DLE) of HCP and FCP from HMs and FMs. Briefly, HMs or FMs were dispersed in DMSO and stirred for $4 \mathrm{~h}$. After filtration through a $0.22 \mu \mathrm{m}$ filter, drugs were measured $(245 \mathrm{~nm}$, flow rate: $1.0 \mathrm{~mL} / \mathrm{min}, 30^{\circ} \mathrm{C}$ ). The standard curves of HCP and FCP were drawn in advance. DLC and DLE were counted according to the following formula:

$$
\begin{aligned}
D L E \%= & \frac{\text { Amount of drugs entrapped in micelles }}{\text { Initial amount of drug added }} \\
& \times 100 \%
\end{aligned}
$$

$$
\begin{aligned}
D L C \%= & \frac{\text { Amount of drugs entrapped in micelles }}{\text { Total amount of micelles }} \\
& \times 100 \%
\end{aligned}
$$

Size distribution and morphology of micelles were detected by dynamic light scattering (DLS) on a Zetasizer Nano ZS (Malvern Instrument Ltd., Malvern, UK) and transmission electron microscopy (TEM) on JEM-2100F (Tokyo, Japan). Briefly, micelles and core cross-linked micelles were dispersed in pure water. TEM samples were prepared by the typical method. A drop of micelle suspension was placed on the carbon film copper mesh for 5 min. Finally, the suspension was removed and the samples were dried at room temperature for 24 $h$ before TEM measurement.

\section{Buffer Capacity}

The buffering capacity of the CMs and control micelles (mPEG-lys(Ce6)-PBLG) was investigated according to a previously published method. ${ }^{21}$ Both types of micelle aqueous solutions $(0.5 \mathrm{mg} / \mathrm{mL})$ were prepared and the $\mathrm{pH}$ was adjusted to 10 using $\mathrm{NaOH}(1 \mathrm{~mol} / \mathrm{L})$. The micellar suspension was titrated with $0.1 \mathrm{~mol} / \mathrm{L} \mathrm{HCl}$ solution and the $\mathrm{pH}$ of the obtained mixture was recorded continuously. The titration curve was obtained via plotting the system $\mathrm{pH}$ against the volume of $\mathrm{HCl}$ solution.

\section{Stimuli-Responsiveness}

To demonstrate the $\mathrm{pH}$-responsivity, size distribution and Zeta potential of BMs and CMs were analyzed by DLS. mPEG-lys (Ce6)-PBLG micelles were used as control group. Briefly, micelles $(0.5 \mathrm{mg} / \mathrm{mL})$ were measured in PBS solutions at $\mathrm{pH} 7.4,6.8$, and 5.5. Micelle size distribution and Zeta potential were assessed by DLS, and micelle morphology was assessed by TEM. To further 
evaluate the cross-linkage of micelles, BMs and CMs were dispersed in DMSO and PBS, respectively. To survey ROS-responsive degradation of micelles, BMs and CMs were incubated in PBS pH 7.4 containing $0,0.1 \mathrm{mM}$ and 1 $\mathrm{mM} \mathrm{H}_{2} \mathrm{O}_{2}$. DLS and TEM record particle size distribution and morphology. For practical observation of this phenomenon, a picture of micelles was taken.

\section{Dilution of Micelles}

The blank mPEG-lys (Ce6)-PGA-AIM micelle was diluted with $0.9 \mathrm{~mL}$ DMSO, and $\mathrm{Zn}^{2+}(0.1 \mathrm{~mL}, 1 \mathrm{mg} / \mathrm{mL})$ was added or not added. Pictures were taken to observe the Tyndall effect. In order to further realize the cross-linking of micelles, the FL spectra of micelles at $400 \sim 800 \mathrm{~nm}$ were recorded. In addition, the particle size distribution and morphology of the diluted micelles were determined by DLS and TEM.

\section{Drug Release}

The dialysis method was performed to detected drug release from FMs or CFMs in vitro. FMs or CFMs $(1 \mathrm{mg} / \mathrm{mL})$ were transferred into a Float-A-Lyzer G2 dialysis device (MWCO $100 \mathrm{KDa}$, Spectrum) and drug release was conducted in $\mathrm{PBS}\left(\mathrm{pH} \mathrm{7.4)}\right.$ ) at $37^{\circ} \mathrm{C}$. At predetermined time points, $1.0 \mathrm{~mL}$ of sample solution was taken out and $\mathrm{Pt}$ concentration was quantified by atomic absorption spectroscopy (AAS). ${ }^{19}$ An equal volume of fresh PBS was immediately replenished. The same drug release procedure was carried out in triplicate in PBS pH 6.5 and $\mathrm{pH} 5.5$ with or without $0,0.1 \mathrm{mM}$ and 1 $\mathrm{mM} \mathrm{H} \mathrm{O}_{2}$, respectively.

\section{Cellular Uptake}

To evaluate the enhanced cellular uptake by slightly acidic microenvironment, SKOV3 cells $\left(1.0 \times 10^{6}\right.$ cells/well $)$ were placed in 6-well plates for $12 \mathrm{~h}$. Then, culture medium of half wells was substituted with adjusted medium ( $\mathrm{pH}$ 6.8, adjusted with $0.1 \mathrm{M} \mathrm{HCl}$ solution). BMs were added into each well and cells were incubated for 0.5 $\mathrm{h}$ and $1 \mathrm{~h}$. After that, cells were washed with PBS, fixed with $4 \%$ formaldehyde for $15 \mathrm{~min}$ and stained with DAPI for $10 \mathrm{~min}$. Coverslips were placed onto glass microscope slides and the fluorescence imaging of cells was analyzed by confocal laser scanning microscopy (CLSM) on a Leica confocal system.

To evaluate the endosome escape of micelles and accumulation of drugs into cells, SKOV3 cells $\left(1.0 \times 10^{6}\right.$ cells/ well) were placed in 6-well plates for $12 \mathrm{~h}$. Then, cells were induced with mPEG-lys (Ce6)-PBLG micelles (negative control, NC) or BMs for $4 \mathrm{~h}, 8 \mathrm{~h}$ and $12 \mathrm{~h}$. After that, cells were stained with Lysotracker Green following the instructions then washed with PBS. And cells were fixed with $4 \%$ formaldehyde for $15 \mathrm{~min}$ and stained with DAPI for $10 \mathrm{~min}$. CLSM was used to analyze the fluorescence imaging of cells by placing the covering membrane on a glass slide.

\section{MTT Assay}

SKOV3 cells $\left(4 \times 10^{3}\right.$ cells/well $)$ were plated in 96-well plates. Oxygen-saturated $\mathrm{CMs}, \mathrm{CHMs}$ and $\mathrm{CPMs}$ were added with equivalent $\mathrm{Pt}$ concentrations ranging from 0.05 to $1.6 \mu \mathrm{M}$. Then the light irradiation was applied for $10 \mathrm{~min}\left(660 \mathrm{~nm}, 100 \mathrm{~mW} / \mathrm{cm}^{2}\right)$. After incubation for $24 \mathrm{~h}$, the cell viability was assessed by the standard 3-(4, 5-dimethylthiazol-2-yl)-2, 5-diphenyltetrazolium bromide (MTT) assay. Cell viability of cells treated with $\mathrm{CMs}$, CHMs and CPMs without light irradiation was evaluated by the same method. Cell viability was counted according to the following formula:

$$
\text { Cell viability } \%=\frac{O D_{\text {sample }}-O D_{\text {blank }}}{O D_{\text {control }}-O D_{\text {blank }}} \times 100 \%
$$

\section{Cell Apoptosis}

Cell apoptosis was detected by flow cytometry. Firstly, the cells stimulated with saline, CMs, CHMs and CPMs with or without light irradiation were collected, resuspended in cold PBS, centrifuged at $1000 \mathrm{rpm}$ in room temperature for 5-10 min. Then, cells were incubated with $5 \mu \mathrm{L}$ AnnexinV-Alexa Fluor 647 for $15 \mathrm{~min}$ and then coincubated with $5 \mu \mathrm{L}$ PI before detection. In the end, the apoptosis rate was tested via flow cytometer (Beckman Coulter, CA, USA).

\section{Animal Model and Biodistribution}

The model of heterogeneous prostate cancer was structured by subcutaneous injection of SKOV3 cells. Mice were randomly divided into three groups $(n=5)$. To visually observe the biodistribution of micelles, saline, free $\mathrm{Ce} 6$ and $\mathrm{CMs}$ were injected via tail vein with $\mathrm{Ce} 6$ amount of $2 \mathrm{mg} / \mathrm{kg}$. After $12 \mathrm{~h}, 24 \mathrm{~h}$ and $48 \mathrm{~h}$, mice of each group were exposed on an infrared imaging system (Caliper, XenoFluor 750) with excitation wavelength at $780 \mathrm{~nm}$. Afterwards, mice were euthanized and tissues, including heart, liver, spleen, lung, kidney and tumor, were harvested for imaging ex vivo. The Institutional Animal 
Care and Use Committee of Tianjin Medical University Cancer Institute and Hospital authorized this research.

\section{Drug Administration}

6-week-old male nude mice were inoculated subcutaneously in the right flank with SKOV3 cells $\left(1.5 \times 10^{6}\right)$. When the xenografts tumor volume grew to an appropriate size successfully, mice were divided into 7 groups $(n=5)$ and treated with different drugs including saline, CMs, CHMs and CFMs with or without light irradiation (Pt: $10 \mu \mathrm{M} / \mathrm{kg}$ ). The body weight and tumor size were recorded every 2 days. Besides, after mice were sacrificed, tissues including heart, liver, spleen, lung, kidney and tumor were collected and fixed for HE and TUNEL staining.

\section{Results and Discussion Characterization of Polymers}

The synthesis procedure of polymers is depicted in Figure 2. The ${ }^{1} \mathrm{H}$ NMR spectra of polymers are shown in Figure 3AF. The peak a $(1.37 \mathrm{ppm})$ and $\mathrm{b}(7.92-7.31 \mathrm{ppm})$ labeled in Figure $3 \mathrm{~A}$ were attributed to the typical peak of Boc and Fmoc, respectively. After deprotection, peak b disappeared, while peak a was still there, which proves the successful synthesis of mPEG-lys(Boc) (Figure 3B). After ringopening polymerization, the signals at $7.26 \mathrm{ppm}$ and 5.07 ppm (peak $\mathrm{d}$ and c) were observed and attributed to the phenyl and methylene groups, respectively (Figure 3C). The numbers of repeated units of the hydrophobic blocks in mPEG-lys(Boc)-PBLG were calculated to be 38 on average from the spectra of ${ }^{1} \mathrm{H}$ NMR by comparing the integral area of PEG segments and benzene groups. Thus, the molecular weight $\left(M_{\mathrm{n}}\right)$ of mPEG-lys(Boc)-PBLG was $13,550 \mathrm{~g} /$ mol. mPEG-lys(Boc)-PBLG was successfully de-protected, proved by the disappearance of peak a as shown in Figure 3D. Ce6 was successfully conjugated as shown in Figure 3E. After aminolysis, both peak $\mathrm{c}$ and d disappeared and emerging peaks ( $\mathrm{f}, \mathrm{g}$ and $\mathrm{h}$ ) appeared that were attributed to imidazoles (7.63 ppm, $7.15 \mathrm{ppm}$ and $6.88 \mathrm{ppm}$ ), while peak e can still be observed (Figure 3F).

\section{Characterization of Micelles}

To evaluate $\mathrm{pH}$-responsive size distribution, mPEG-lys (Ce6)-PBLG micelles, BMs and CMs were exposed on buffer solution of $\mathrm{pH} 7.4,6.8$ and 5.5. As shown in Figure 4A, mPEG-lys(Ce6)-PBLG micelles exhibited narrow size distribution with average size of $231.6 \pm 9.8 \mathrm{~nm}$ and $\mathrm{pH}$ values did not change the size distribution.
However, size distribution of BMs changed with $\mathrm{pH}$ values as shown in Figure 4B. BMs exhibited average size of $169.7 \pm 6.2 \mathrm{~nm}$ in $\mathrm{pH}$ 7.4. It increased to $243.9 \pm$ $10.3 \mathrm{~nm}$ in $\mathrm{pH} 6.8$ and $265.1 \pm 13.7 \mathrm{~nm}$ in $\mathrm{pH} 5.5$. Furthermore, significantly broadened size distribution was observed in $\mathrm{pH} 6.8$ and 5.5, which was further proved by the TEM photos of BMs in different $\mathrm{pH}$ values (Figure 4E). Changes of size distribution of BMs were caused by the protonation of imidazoles. CMs exhibited decreased average size of $113.7 \pm 4.8 \mathrm{~nm}$ in $\mathrm{pH} 7.4$ compared with BMs, which was possibly caused by the cross-linkage. Besides, size increased with decreasing $\mathrm{pH}$, as shown in Figure 4C.

To demonstrate the cross-linkage, BMs and CMs were dispersed in water or DMSO and the corresponding size distribution was measured as shown in Figure 4D. BMs in water exhibited larger size than CMs in water. $\mathrm{CMs}$ in DMSO exhibited broadened size distribution, while no signal was detected when BMs were dispersed in DMSO. To further evaluate the long-term stability, the micelles were dispersed in water, PBS and FBS and incubated at $37{ }^{\circ} \mathrm{C}$ for 12 days. The particle size changes of the micelles were detected using DLS. The results showed that the size of the micelles did not change significantly in the water. It shows that micelles have the most stable structure in water and can be used as the first choice during long-term storage (Figure S1). Additionally, the corresponding size of $\mathrm{CMs}$, CHMs and CPMs were also measured as shown in Figure S2.

\section{ROS-Responsiveness}

mPEG-lys(Ce6)-PBLG micelles, BMs and CMs were incubated with $1 \mathrm{mM} \mathrm{H}_{2} \mathrm{O}_{2}$. Corresponding size distribution was demonstrated in Figure 5. Average size of mPEGlys(Ce6)-PBLG micelles fluctuated around $180 \mathrm{~nm}$, indicating good stability during incubation for $14 \mathrm{~h}$ (Figure 5A). CMs can keep stable during the first $6 \mathrm{~h}$, while fast degradation occurs during the next $8 \mathrm{~h}$ (Figure 5B). BMs exhibited a similar degradation progress (Figure 5C). Interestingly, BMs kept stable during the first $4 \mathrm{~h}$, indicating faster degradation than CMs. Figure 5D demonstrated the size distribution of $\mathrm{BMs}$ and $\mathrm{CMs}$ after $\mathrm{H}_{2} \mathrm{O}_{2}$ treatment. As Figure 5E shows, no particular morphology can be observed in TEM photo of BMs after $\mathrm{H}_{2} \mathrm{O}_{2}$ treatment, indicating complete degradation. While, degradation still occurred after CMs were treated with $\mathrm{H}_{2} \mathrm{O}_{2}$ and sphericallike morphology still can be observed in Figure 5F. 


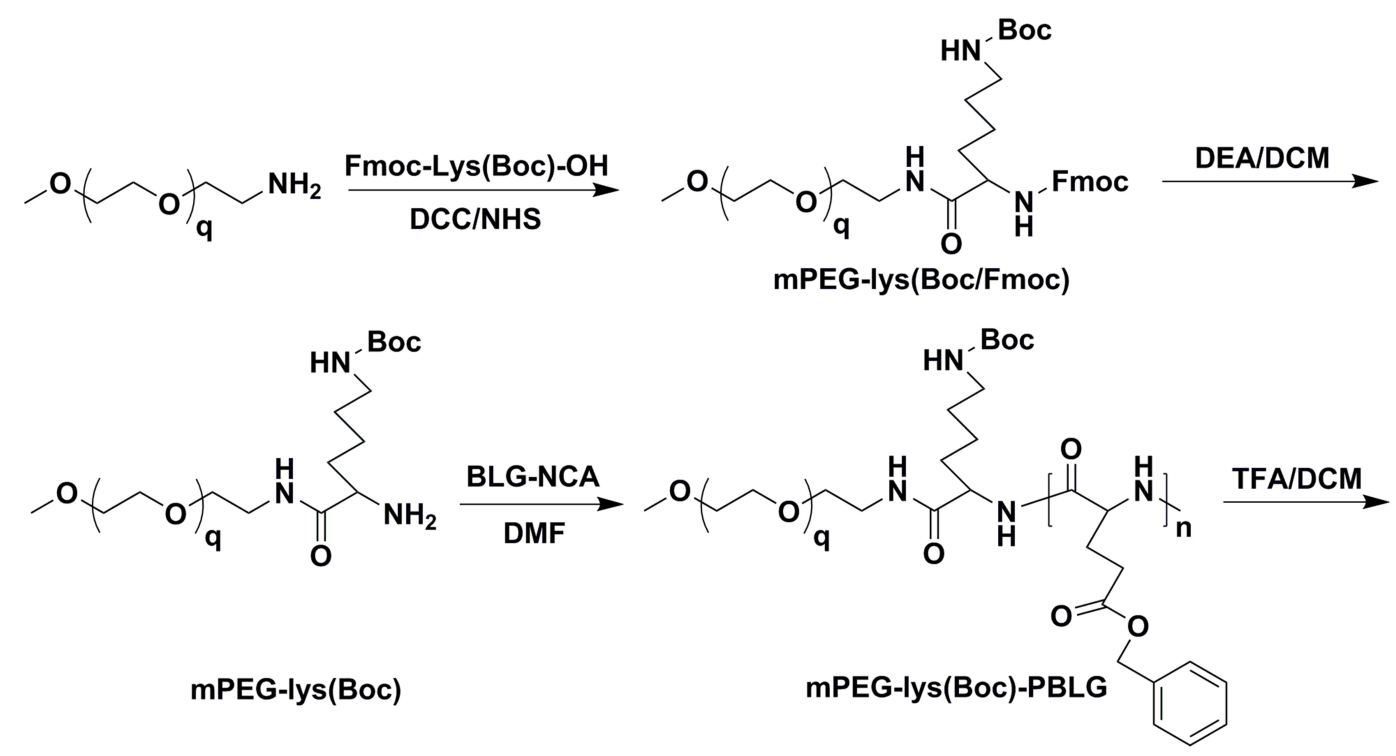

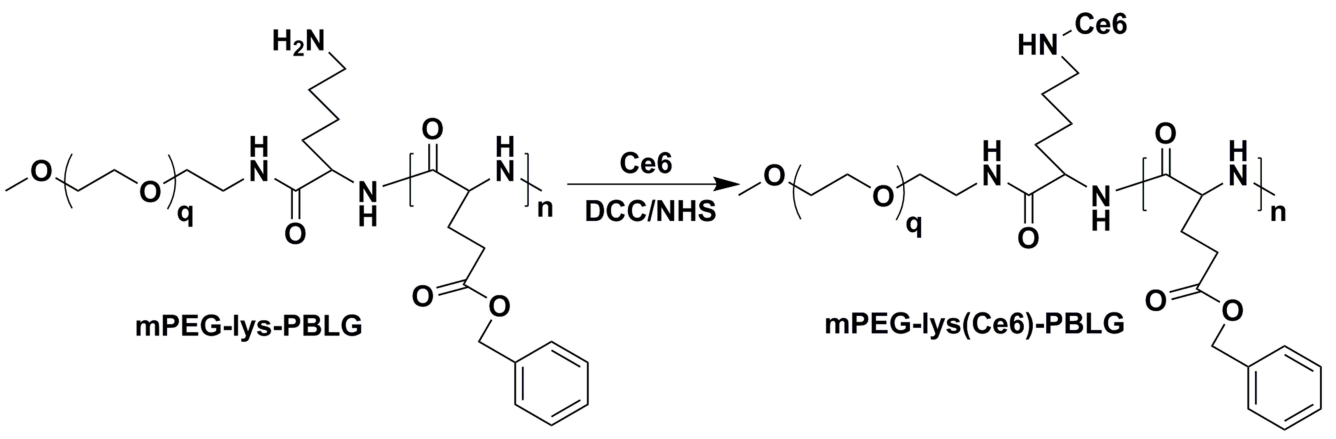

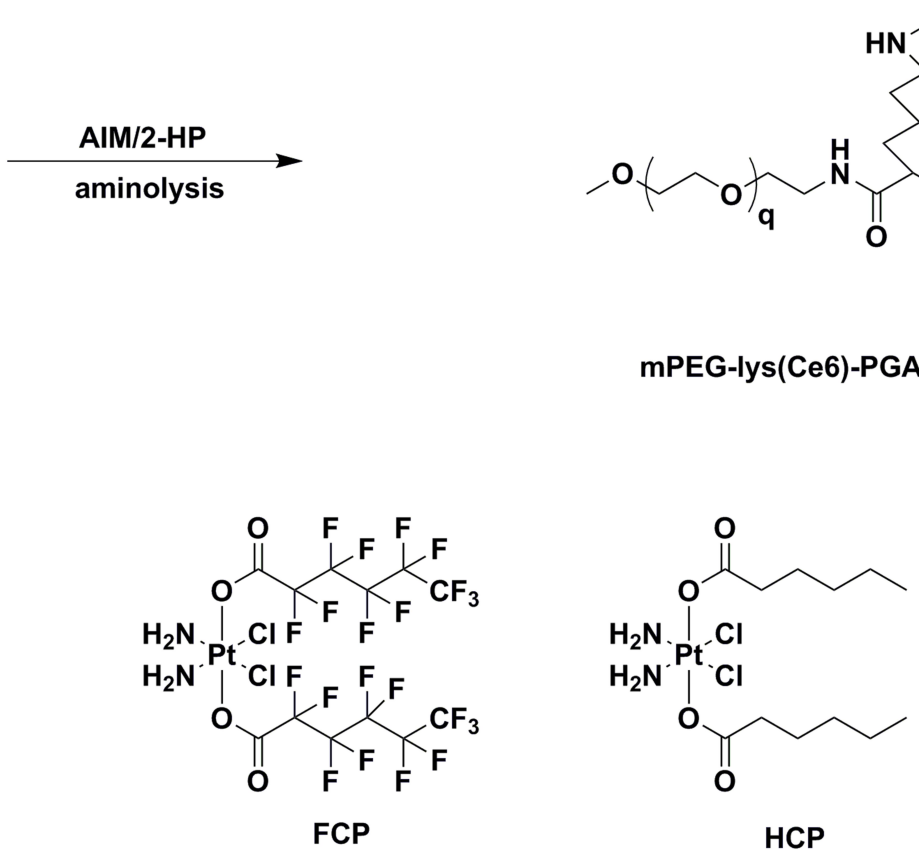

Figure 2 Synthesis scheme of polymers. 
Chen et al

Dovepress
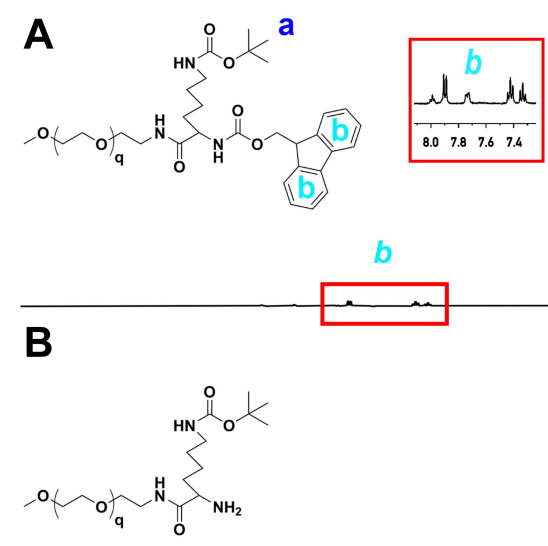

D
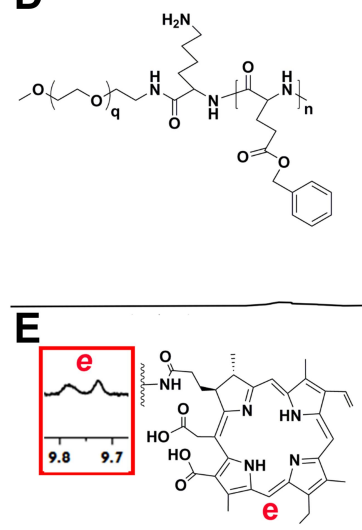

F

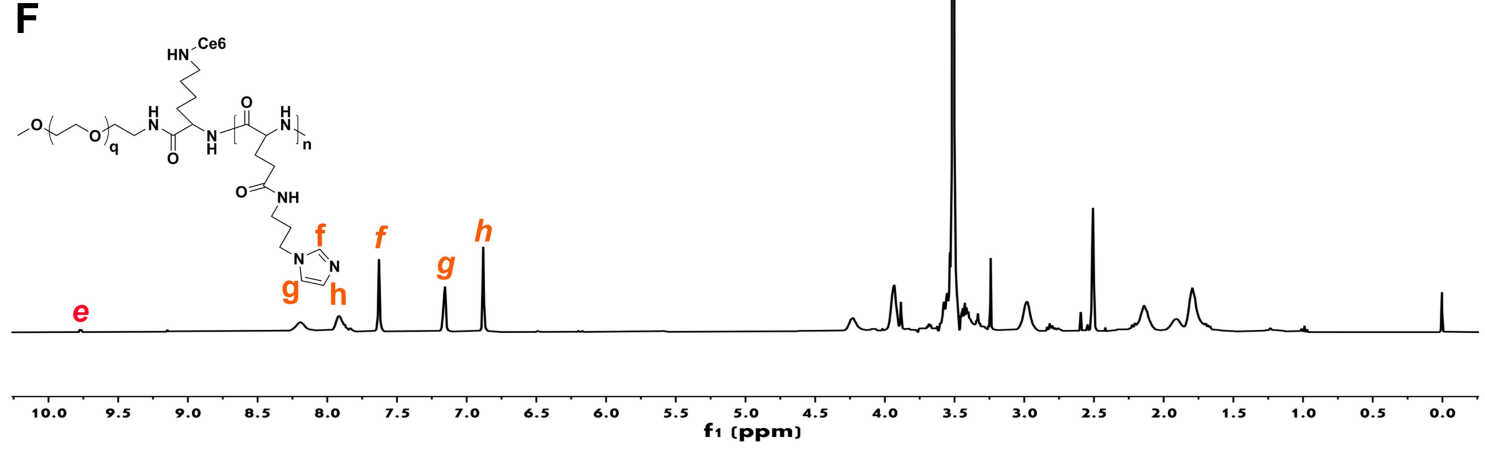

Figure 3 'H NMR of polymers in DMSO-d 6 : mPEG-lys(Boc/Fmoc) (A); mPEG-lys(Boc) (B); mPEG-lys(Boc)-PBLG (C); mPEG-lys-PBLG (D); mPEG-lys(Ce6)-PBLG (E); and mPEG-lys(Ce6)-PGA-AIM (F).

3686

https://doi.org/| 0.2147//JN.S298|46

DovePress

International Journal of Nanomedicine 2021:16 

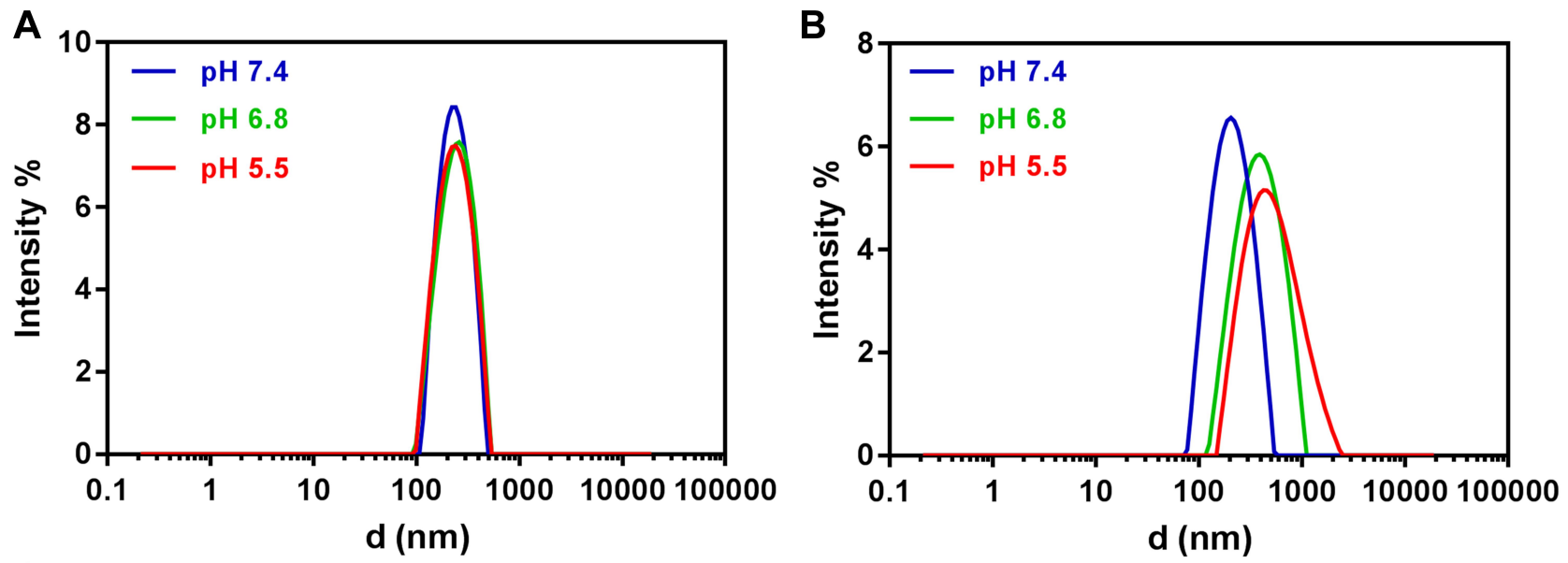

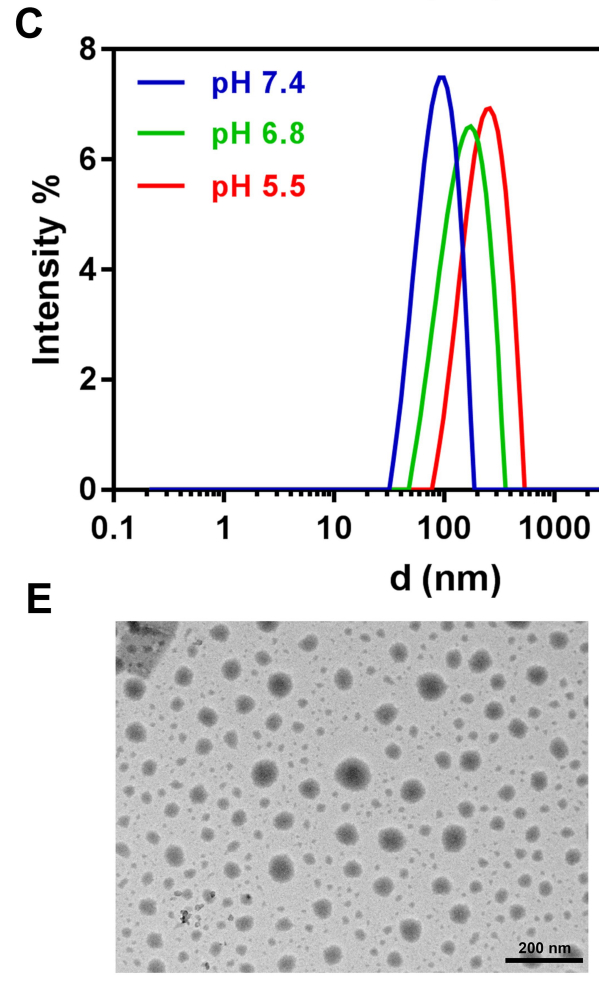

pH 7.4

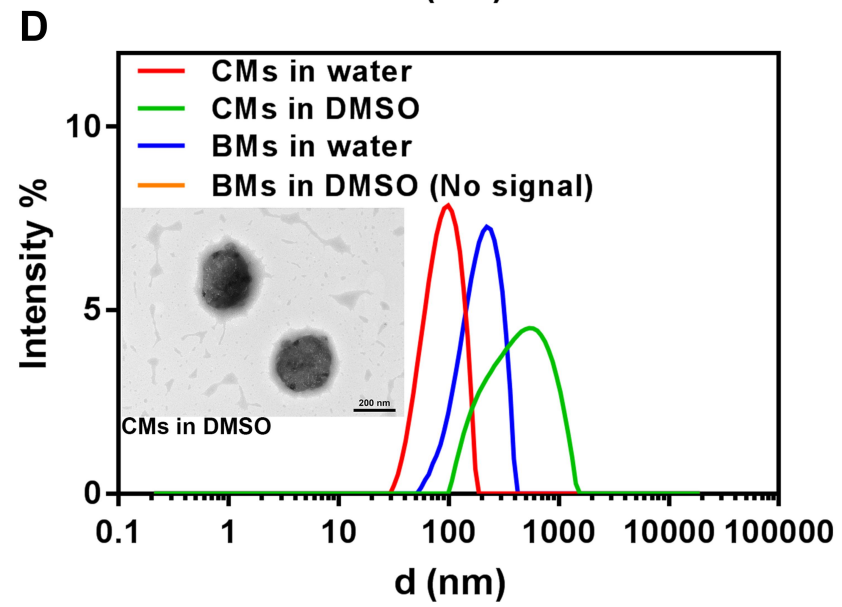

10000100000

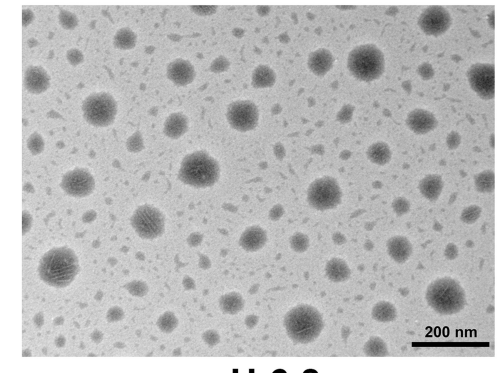

pH 6.8

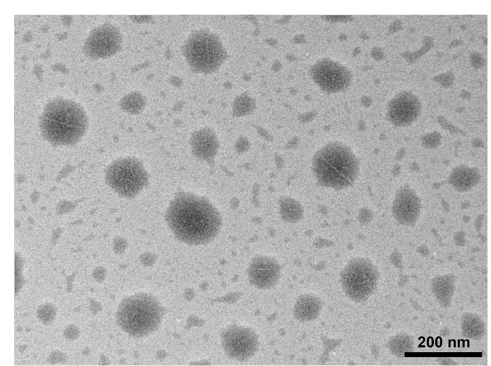

pH 5.5

Figure 4 Size distribution of micelles in buffer solution of $\mathrm{pH}$ 7.4, 6.8 and 5.5: mPEG-lys(Ce6)-PBLG micelles (A); BMs (B); and CMs (C). Size distribution of BMs and CMs in water or DMSO (D). The inserted picture was TEM photo of CMs in DMSO. Morphology of BMs in different $\mathrm{pH}$ values (E).

\section{Drug Release}

Drug release from FCP-loaded mPEG-lys(Ce6)-PBLG micelles, FMs and CFMs was investigated in different conditions. To evaluate the influence of $\mathrm{pH}$ values on drug release, mPEG-lys(Ce6)-PBLG micelles, FMs and CFMs were dialyzed against buffer solution of $\mathrm{pH} 7.4$, 6.8 and 5.5. As shown in Figure 6A, at pH 7.4, all micelles exhibited rather slow drug release with cumulative drug release of $48 \mathrm{~h}$ less than $20 \%$. Among these drug-loaded micelles, CFMs exhibited slower drug release than FMs, due to the cross-linkage of CFMs, facilitating prevention of premature release of encapsulated drugs during blood circulation. When $\mathrm{pH}$ decreased to 6.8 drug release of FMs was dramatically enhanced with cumulative amount of drugs $26.9 \%$ for $48 \mathrm{~h}$, while FCP-loaded mPEG-lys(Ce6)PBLG micelles and CFMs at $\mathrm{pH} 6.8$ exhibited similar drug release profiles with those at $\mathrm{pH} 7.4$ (Figure 6B). With the further reduction of $\mathrm{pH}$ to 5.5 (Figure $6 \mathrm{C}$ ), drug release of 


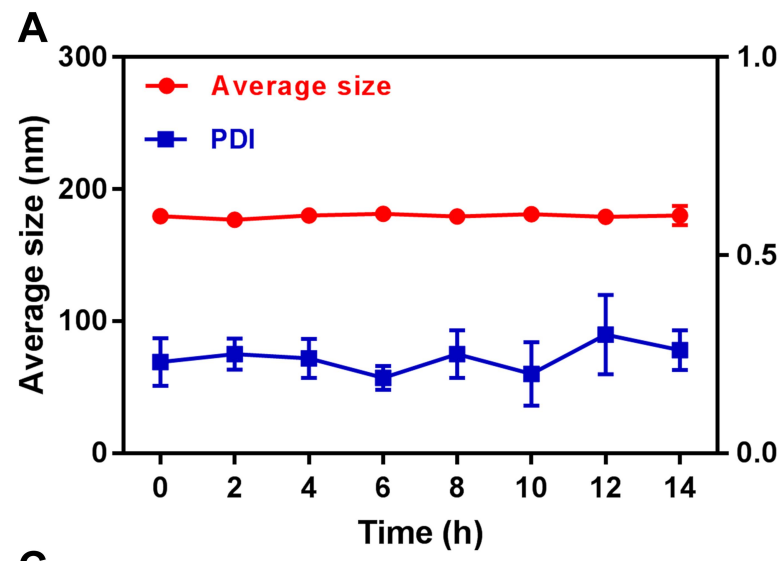

C
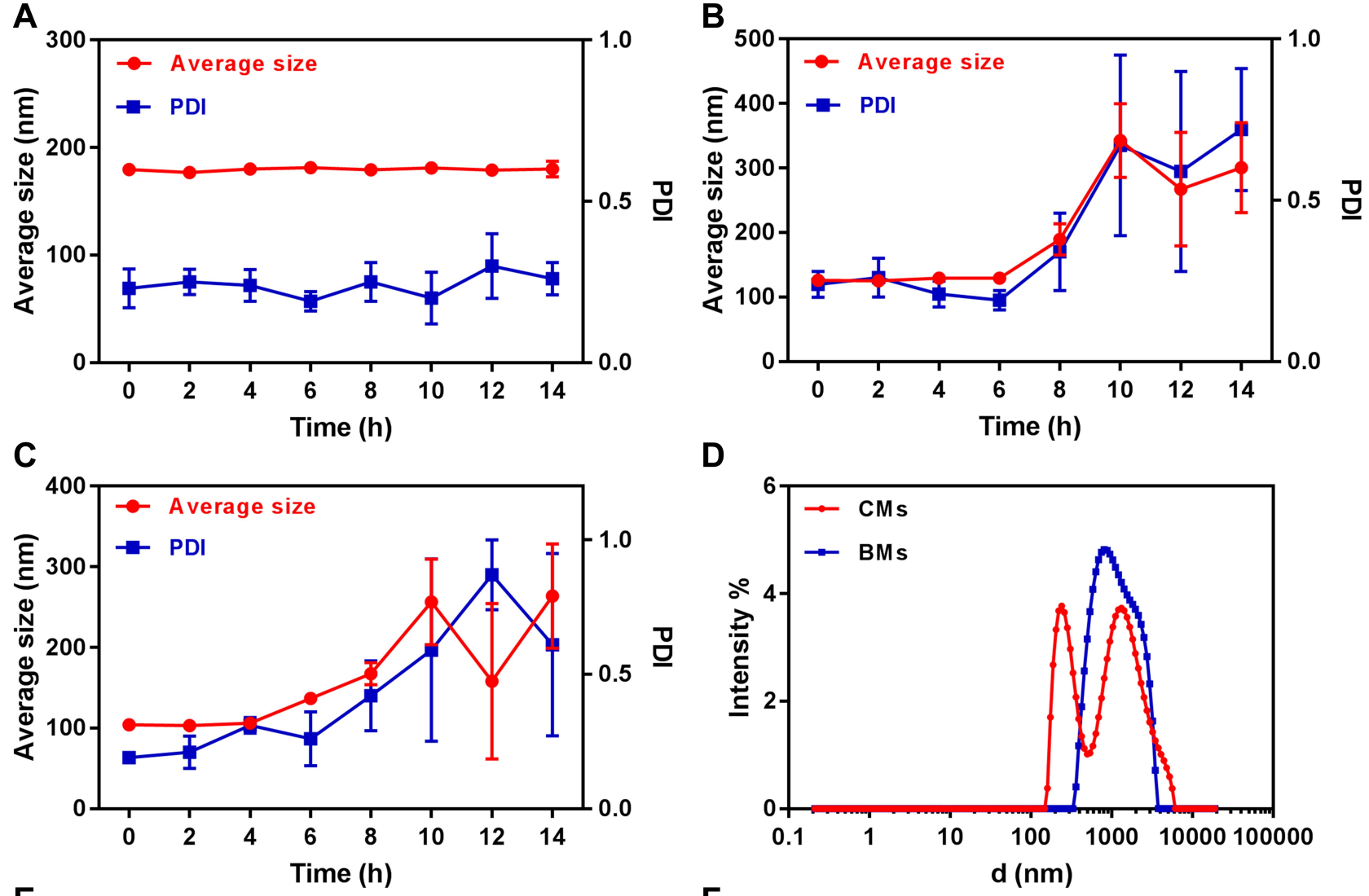

D

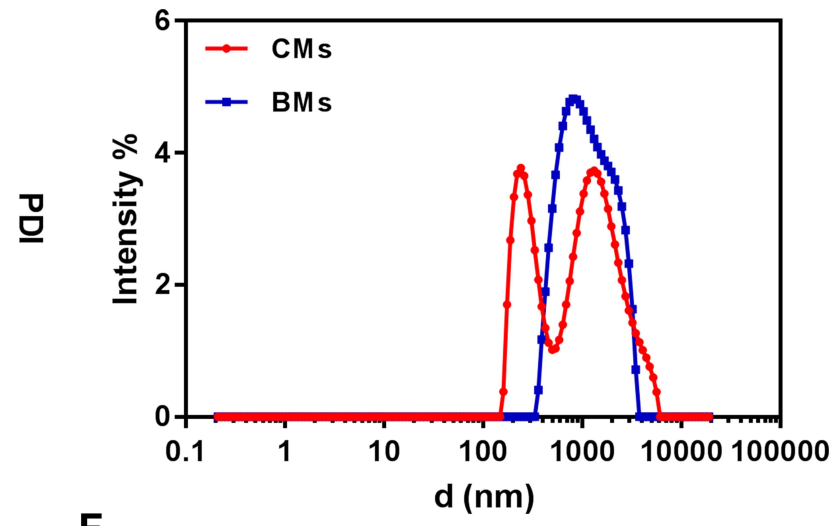

E
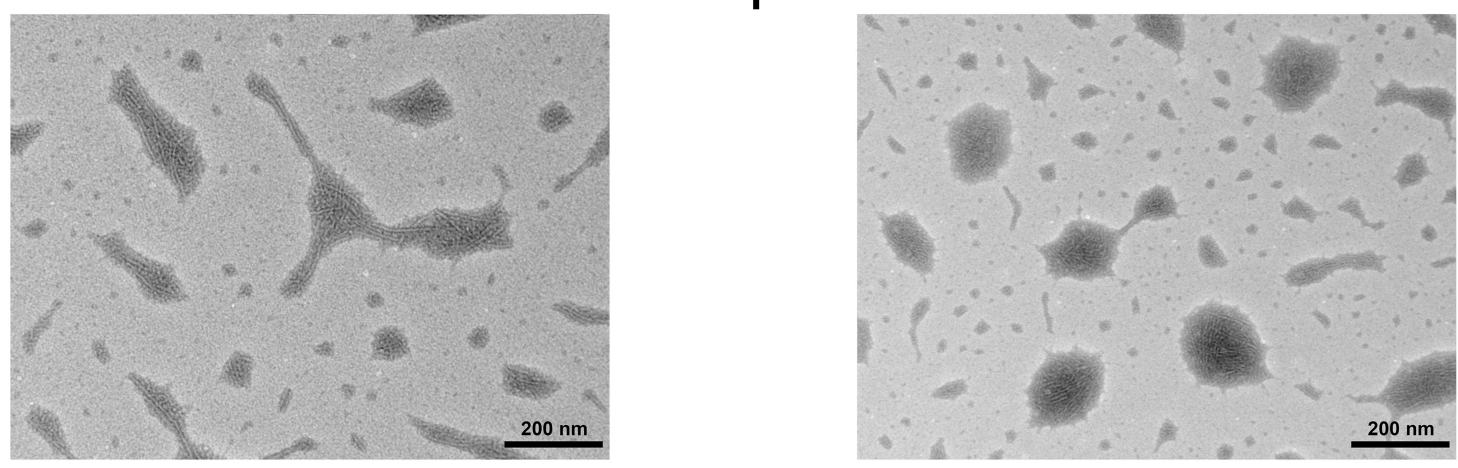

Figure 5 Changes of average size and PDI with time of different micelles: mPEG-lys(Ce6)-PBLG micelles (A); CMs (B); and BMs (C). Size distribution of BMs and CMs after $\mathrm{H}_{2} \mathrm{O}_{2}$ treatment (D). TEM photo of BMs (E) and CMs (F) treated with $\mathrm{H}_{2} \mathrm{O}_{2}$.

FMs and CFMs was rather enhanced than that at $\mathrm{pH} 7.4$ and 6.8, as well as FCP-loaded mPEG-lys(Ce6)-PBLG micelles. Specifically, the cumulative drug release of FCPloaded mPEG-lys(Ce6)-PBLG micelles, FMs and CFMs was $15.6 \%, 69.7 \%$, and $55.1 \%$, respectively. Because of cross-linkage, CFMs exhibited slower drug release than FMs. These results indicated cross-linked mPEG-lys(Ce6)PGA-AIM micelles can encapsulate drugs efficiently during blood circulation and extracellular tumor cells, while achieving fast drug release in endosome/lysosomes in tumor cells. To evaluate the effects of ROS on drug release, drug-loaded micelles were incubated with $0.1 \mathrm{mM}$ $\mathrm{H}_{2} \mathrm{O}_{2}$ and $1 \mathrm{mM} \mathrm{H} \mathrm{H}_{2} \mathrm{O}_{2}$. As shown in Figure 6D, drug release from FCP-loaded mPEG-lys(Ce6)-PBLG micelles in $0.1 \mathrm{mM} \mathrm{H}_{2} \mathrm{O}_{2}$ condition was similar with that in $1 \mathrm{mM}$ $\mathrm{H}_{2} \mathrm{O}_{2}$, with cumulative drug release amount less than $16 \%$ for $48 \mathrm{~h}$. Both FMs and CFMs exhibited faster drug release than FCP-loaded mPEG-lys(Ce6)-PBLG micelles. And drug release from micelles in $1 \mathrm{mM} \mathrm{H}_{2} \mathrm{O}_{2}$ was enhanced compared with that in $0.1 \mathrm{mM} \mathrm{H}_{2} \mathrm{O}_{2}$. For example, $92.0 \%$ of drugs were released from FMs in $1 \mathrm{mM}$ $\mathrm{H}_{2} \mathrm{O}_{2}$ for $48 \mathrm{~h}$, while $74.1 \%$ were released in $0.1 \mathrm{mM}$ 

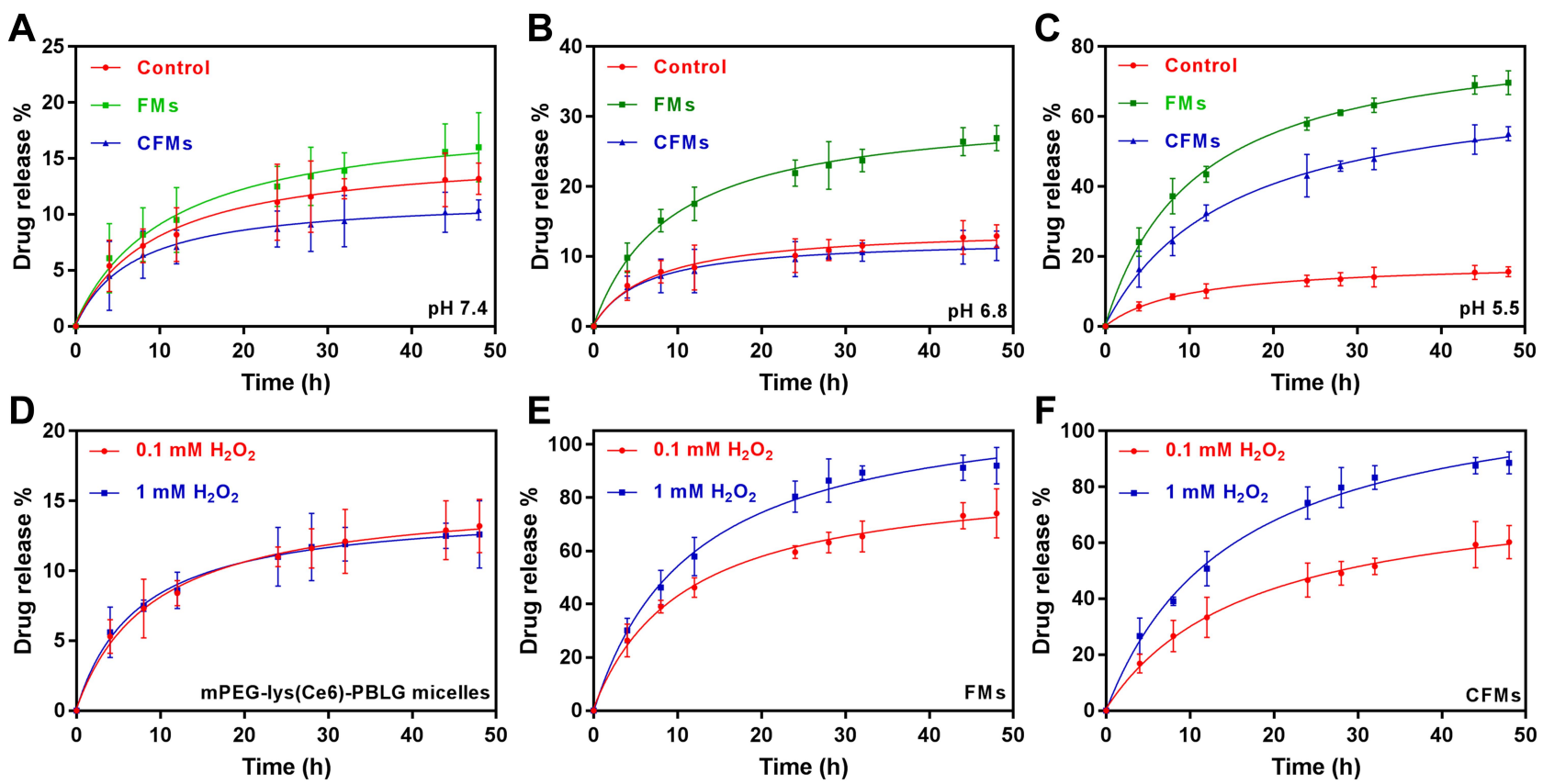

Figure 6 Drug release from FCP-loaded mPEG-lys(Ce6)-PBLG micelles, FMs and CFMs at different conditions: $\mathrm{pH} 7.4(\mathbf{A})$, $\mathrm{pH} 6.8(\mathbf{B})$ and $\mathrm{pH} 5.5$ (C). Drug release at conditions of $\mathrm{pH} 7.4$ with $0.1 \mathrm{mM} \mathrm{H}_{2} \mathrm{O}_{2}$ or $\mathrm{I} \mathrm{mM} \mathrm{H}_{2} \mathrm{O}_{2}$ from different micelles: FCP-loaded mPEG-lys(Ce6)-PBLG micelles (D), FMs (E) and CFMs (F).

$\mathrm{H}_{2} \mathrm{O}_{2}$ (Figure 6E). Interestingly, the difference of drug release between $0.1 \mathrm{mM} \mathrm{H}_{2} \mathrm{O}_{2}$ and $1 \mathrm{mM} \mathrm{H}_{2} \mathrm{O}_{2}$ was more obvious. $88.6 \%$ of drugs were released from CFMs in $1 \mathrm{mM} \mathrm{H}_{2} \mathrm{O}_{2}$ for $48 \mathrm{~h}$, while $60.3 \%$ were released in 0.1 $\mathrm{mM} \mathrm{H} \mathrm{H}_{2} \mathrm{O}_{2}$ (Figure $6 \mathrm{~F}$ ). The difference mainly resulted from cross-linkage of CFMs. More importantly, crosslinkage did not restrain drug release and ROS-triggered drug release was achieved. In addition, we detected the changes in ROS levels before and after PDT (Figures S5 and S6).

\section{Cellular Uptake and Cytotoxicity}

To evaluate the influence of $\mathrm{pH}$ values on cellular uptake of $\mathrm{pH}$-responsive micelles, SKOV3 cells were treated with mPEG-lys(Ce6)-PBLG or mPEG-lys(Ce6)-PGA-AIM micelles in adjusted culture media of $\mathrm{pH} 7.4$ and $\mathrm{pH} 6.8$. As shown in Figure 7A, almost no difference of $\mathrm{Ce} 6$ fluorescence was observed after cells were treated with mPEG-lys(Ce6)-PBLG micelles. However, cells treated with mPEG-lys(Ce6)-PGA-AIM micelles at $\mathrm{pH} \quad 6.8$ showed increased $\mathrm{Ce} 6$ fluorescence compared with that at $\mathrm{pH}$ 7.4. To further quantitatively measure the internalized amount of micelles, mean fluorescence intensity of cells treated with different micelles at $\mathrm{pH} 7.4$ and $\mathrm{pH} 6.8$ was detected as shown in Figure 7B. Cells with pretreatment at both $\mathrm{pH}$ values exhibited significantly increased fluorescence compared with cells without treatment. Cells at $\mathrm{pH} 6.8$ treated with mPEG-lys(Ce6)-PBLG micelles showed slightly increased fluorescence compared with these at pH 7.4, which is possibly caused by the enhanced permeability of cell membrane at lower $\mathrm{pH}$ values. However, cells at pH 6.8 treated with mPEG-lys(Ce6)PGA-AIM micelles showed significantly increased fluorescence compared with these at $\mathrm{pH} 7.4$, which is supported by the results of Zeta potential in Figure S3.

To further evaluate the ability of endosome/lysosome escape, cells were treated with mPEG-lys(Ce6)-PBLG or mPEG-lys(Ce6)-PGA-AIM micelles and labeled with Lysotracker Green. As shown in Figure 7C, increased Ce6 fluorescence of cells was observed after cells were treated with both micelles, indicating time-dependent intracellular accumulation of micelles. Also, it was worth noting that mPEG-lys(Ce6)-PGA-AIM micelles led to enhanced fluorescence compared with mPEG-lys(Ce6)PBLG micelles after cells were co-incubated for $30 \mathrm{~min}$ and $60 \mathrm{~min}$, which is mainly caused by the expansion of mPEG-lys(Ce6)-PGA-AIM micelles to further relieve aggregation induced quenching (ACQ) of Ce6. ${ }^{22}$ Entrapped micelles in endosome/lysosomes showed yellow regions in merged photos. A large area of overlap of Lysotracker Green stained endosome/lysosomes and mPEG-lys(Ce6)-PBLG micelles can be observed after 

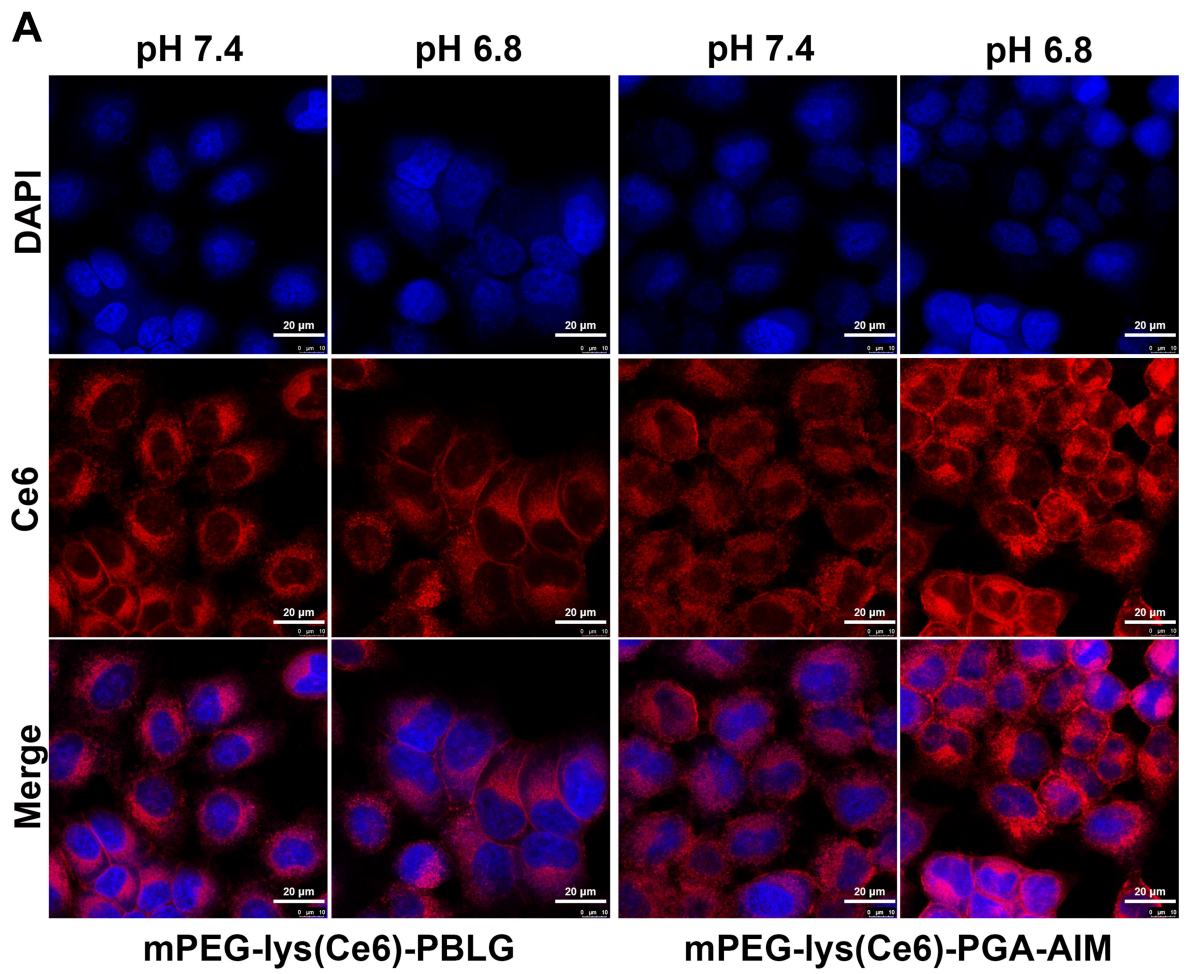

B
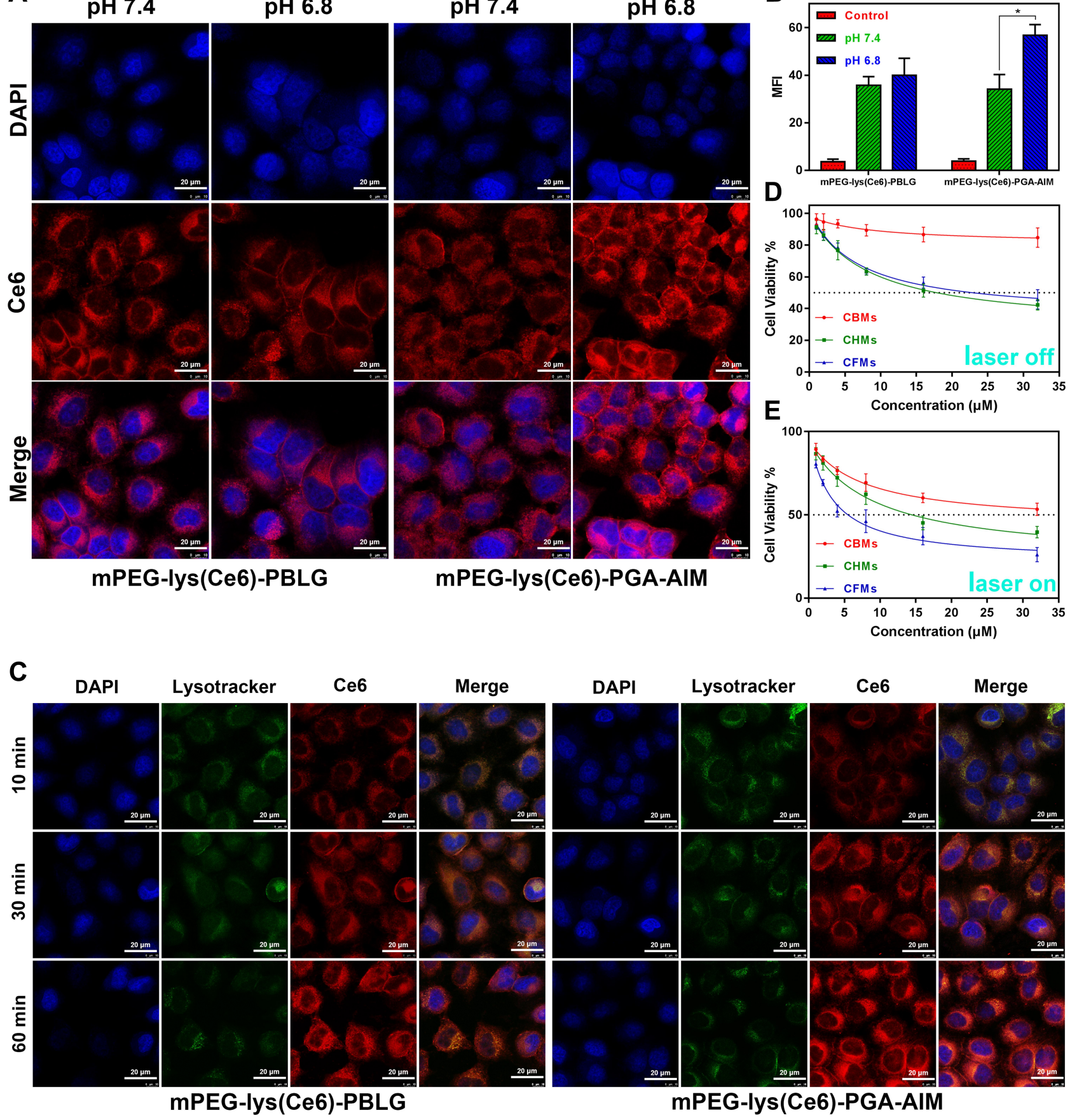

Figure 7 CLSM images (A) and quantitative analysis of fluorescence intensity (B) of SKOV3 cells cultured in medium of $\mathrm{pH} 7.4$ or $\mathrm{pH} 6.8$ treated with mPEG-lys(Ce6)-PBLG or mPEG-lys(Ce6)-PGA-AIM micelles for I h. Intracellular location of mPEG-lys(Ce6)-PBLG or mPEG-lys(Ce6)-PGA-AIM micelles observed by CLSM (C). The late endosomes and lysosomes were stained by Lysotracker Green. Cytotoxicity of CMs, CHMs and CFMs without irradiation of light (D) and with light irradiation (E) against SKOV3 cells for $48 \mathrm{~h}$. Scale bar: $50 \mu \mathrm{m}$.

cells were co-incubated for $10 \mathrm{~min}$ and $30 \mathrm{~min}$, while endosome/lysosome escape of mPEG-lys(Ce6)-PBLG micelles was observed after 60 min of incubation. mPEGlys(Ce6)-PGA-AIM micelles exhibited faster endosome/ lysosome escape than mPEG-lys(Ce6)-PBLG micelles. Specifically, obvious endosome/lysosome escape of
mPEG-lys(Ce6)-PGA-AIM micelles was observed after 30 min of incubation

Cytotoxicity of CBMs, CHMs and CFMs against SKOV3 cells with or without laser irradiation was measured by MTT assay (Figure 7D and E). Without laser irradiation, CBMs exhibited lower cytotoxicity than 
A

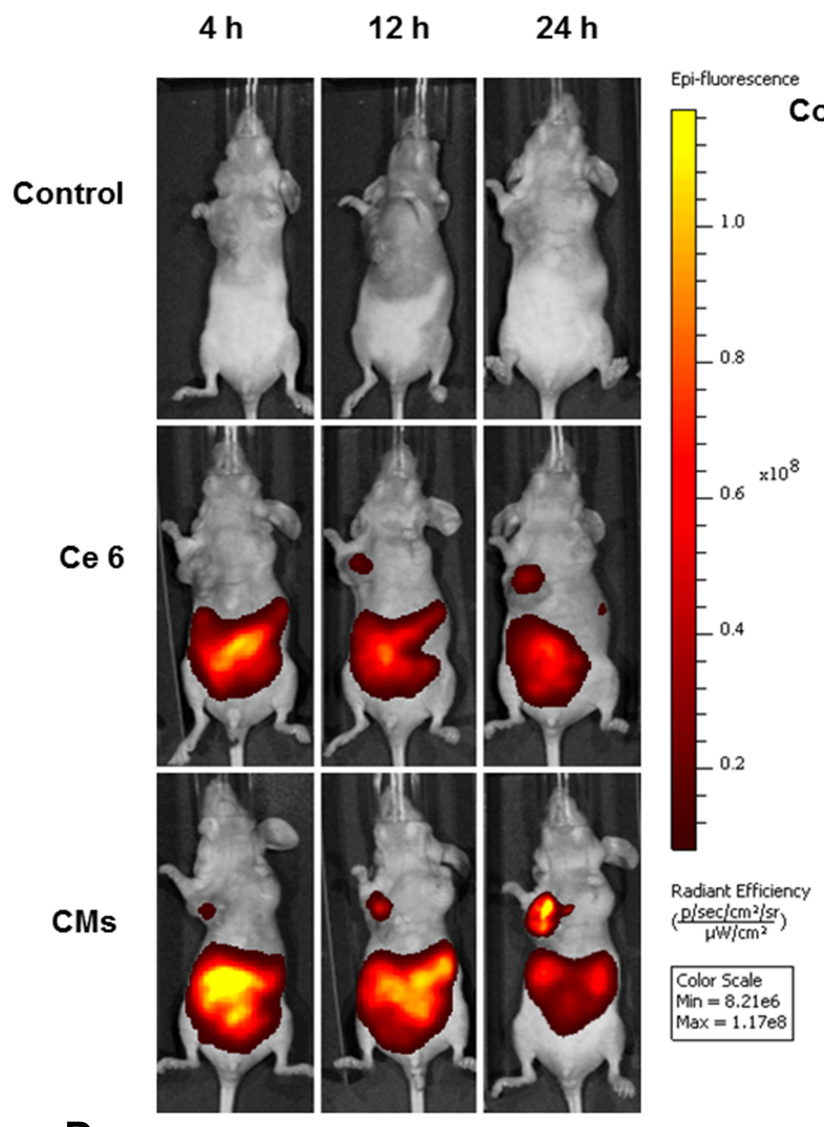

B

Control

Ce 6

CMs

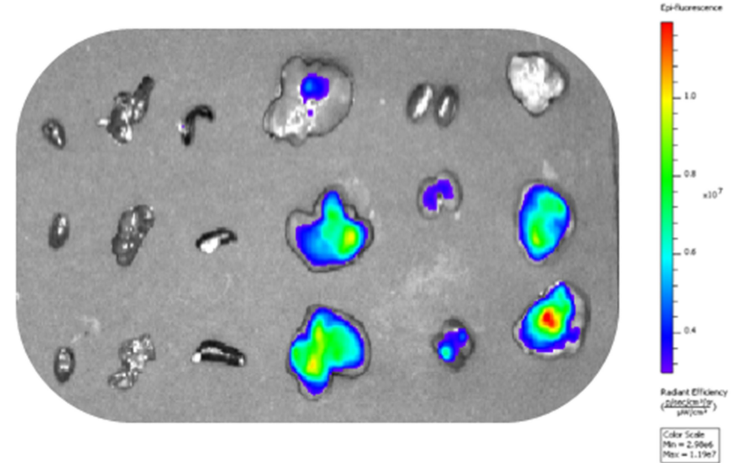

C

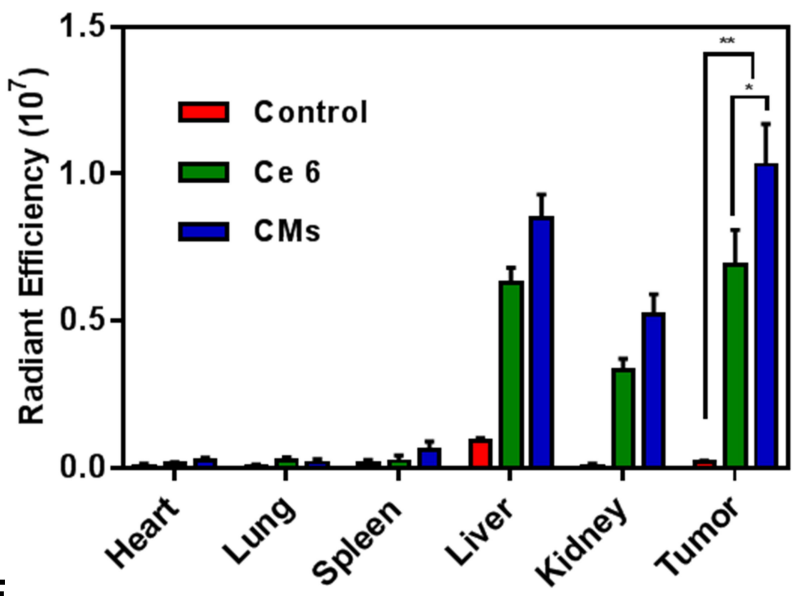

$\mathbf{E}$

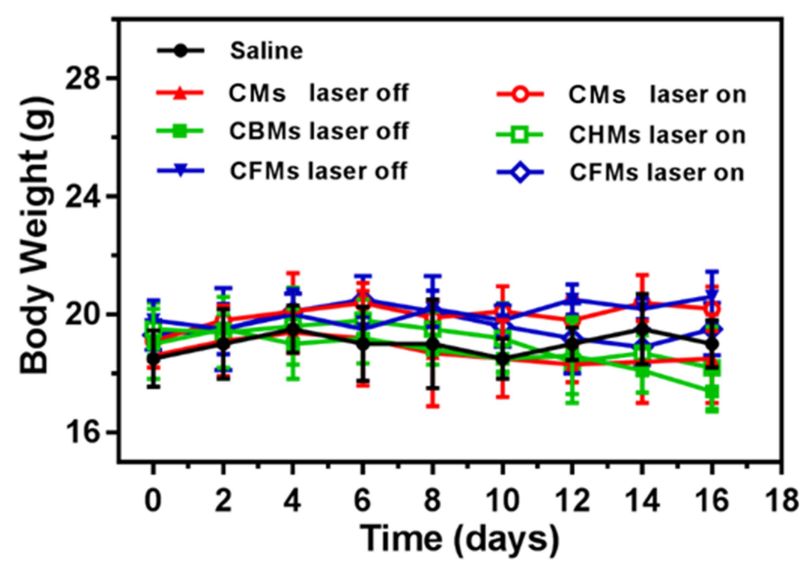

Figure 8 Representative fluorescence images of tumor-bearing mice treated with saline, free Ce6 and CMs for 6 h, 12 h and 24 h (A), and their dissected organs (B) with quantified radiation efficiency $(\mathbf{C})$ at 24 -h post-injection with saline, free $\mathrm{Ce} 6$ and $\mathrm{CMs}$. The tumor volume (D) and body weight change (E) of mice treated with saline, CMs, CHMs and CFMs with or without laser. $* p<0.05, * * p<0.01$, ***p $<0.001$.

CHMs and CFMs. Specifically, cell viability of CBMs was $84.7 \%$, while it was $42.3 \%$ and $45.8 \%$ for CHMs and CFMs, respectively. However, after laser irradiation, all these micelles exhibited decreased cell viability. Cell viability of CBMs was $54.3 \%$, and it was $39.6 \%$ and $26.1 \%$ for CHMs and CFMs, respectively. It is worth noting that
CFMs exhibited comparative cytotoxicity with CHMs without laser irradiation, while significantly increasing cytotoxicity compared with CHMs with laser irradiation. These results directly proved oxygen self-supplied enhanced PDT, which was further proved by the results of cell apoptosis as shown in Figure S4. 


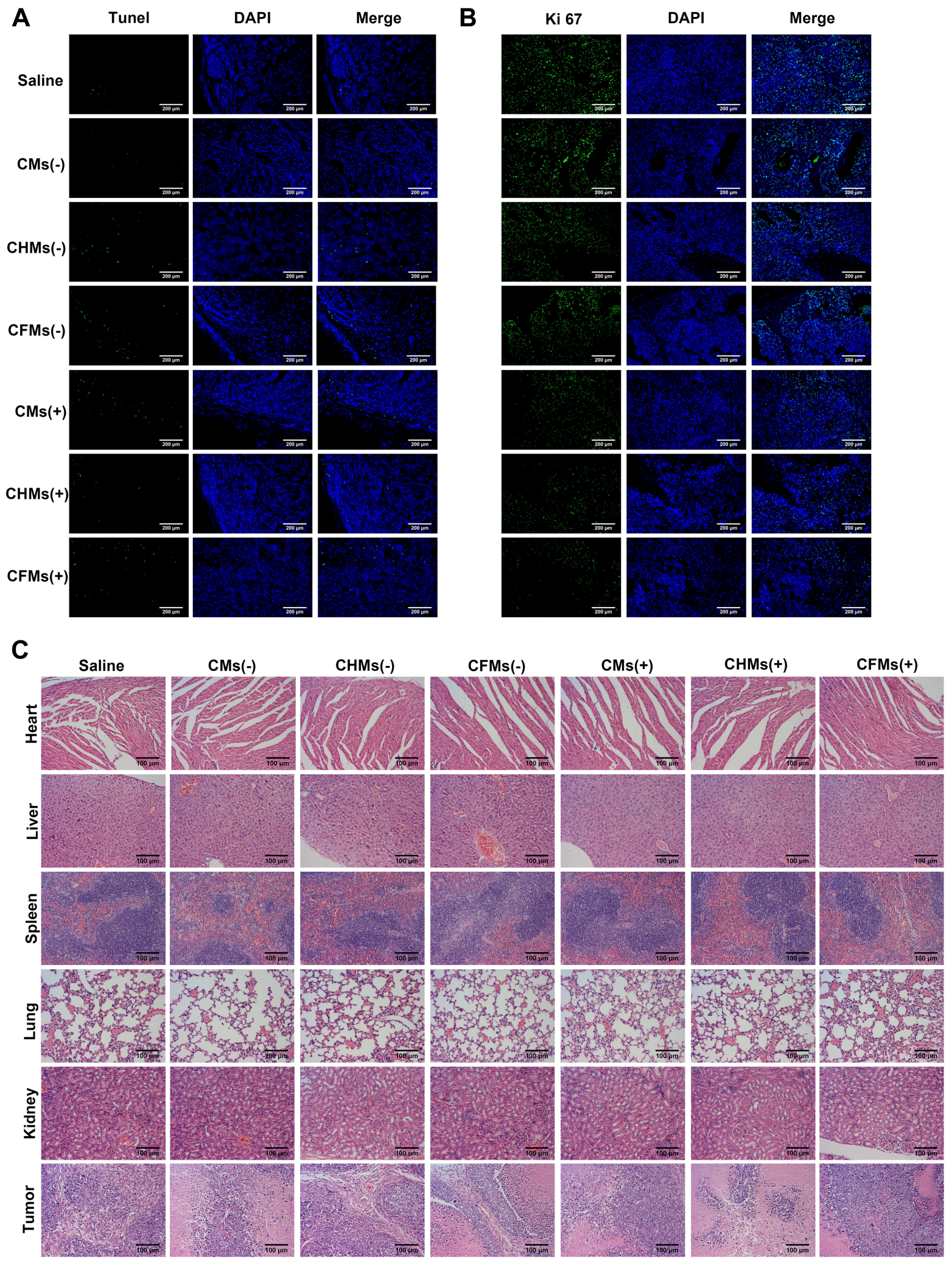

Figure 9 Representative TUNEL (A) and Ki67 (B) immunofluorescence of tumor sections and H\&E staining (C) of various organs from mice stimulated with Saline, CMs, CHMs and CFMs with or without laser, respectively. 


\section{Biodistribution}

To demonstrate the in vivo targeting ability of nanoparticles, mice were treated with saline, free $\mathrm{Ce} 6$ and CMs for $6 \mathrm{~h}, 12 \mathrm{~h}$ and $24 \mathrm{~h}$, and exposed on photographic imaging system. We found that tumors exhibited significant fluorescence signals after CMs were treated for $12 \mathrm{~h}$ (Figure 8A). Enhanced fluorescence signals were observed after injection for $24 \mathrm{~h}$ compared with that of injection for $12 \mathrm{~h}$, indicating time-dependent accumulation of nanoparticles. It was noteworthy that fluorescence signals of free $\mathrm{Ce} 6$ was weak after $12 \mathrm{~h}$ and disappeared after $24 \mathrm{~h}$, suggesting fast metabolism of free Ce6. As shown in Figure 8B, organs and tumors were exposed to observe Ce6 fluorescence after being treated for $24 \mathrm{~h}$. Ce6 fluorescence was mainly located in liver and tumors. In Ce6 imaging, free Ce6 was mainly accumulated in liver and kidney. CMs exhibited higher fluorescence in tumor and lower fluorescence in liver than free $\mathrm{Ce} 6$, indicating enhanced tumortargeting ability of CMs.

\section{In vivo Tumor Therapy}

To evaluate the anticancer effect, tumor-bearing mice were randomly divided into seven groups. Mice were stimulated with saline, Cur, CMs, CHMs and CFMs with or without laser. Firstly, the tumor size of each group was measured every 2 days (Figure 8D). All drug formulations, except $\mathrm{CMs}$ without laser, exhibited significant anticancer effect. Both CHMs and CFMs without laser showed better tumor growth inhibition than $\mathrm{CMs}$ without laser. CHMs and CFMs without laser exhibited the similar anti-tumor effect. However, it was found that CFMs with laser exhibited better antitumor efficiency than any other groups. The loss of body weight induced by all groups was recorded in Figure $8 \mathrm{E}$. These results demonstrated that CFMs with laser exerted great antitumor effect and decreased systemic toxicity compared with CMs and CHMs with laser. Besides, histological analysis of tumors from each group was conducted as shown in Figure 8E. Notably, it was found that CFMs exhibited better anti-tumor effects compared with other groups. The above results confirm that the synergistic photothermal chemotherapy has a highly effective therapeutic effect.

TUNEL and Ki67 immunofluorescence staining of tumor tissue were performed to assess the effects of different groups on apoptosis induction and proliferation inhibition. As shown in Figure 9A and B, CMs, CFMs and CHMs under laser irradiation showed better apoptotic induction and proliferation inhibition than other groups. In the absence of laser irradiation, the inhibition of CFMs and CHMs on cell proliferation was indistinctive, but the induction of apoptosis was more obvious. Notably, CFMs with laser exhibited the most significant anti-tumor effect on tumor. Moreover, similar conclusions were summarized from HE staining results of tumor sections.

Besides, H\&E staining of various organs was further used to evaluate the systemic toxicity of CMs, CFMs and CHMs on mice in vivo. As shown in Figure 9C, the effects of each group on the mice's heart, spleen, lungs and kidneys were mild, regardless of the laser exposure. Liver damage was observed in the CHMs and CFMs groups, indicating that acid-modified Cisplatin (HCP) and perfluorohexanoic acid (FCP) exerted a certain cumulative liver toxicity.

\section{Conclusion}

In conclusion, a novel theranostic agent based on PDT and chemotherapy, namely CFMs was successfully synthesized and drug-loaded core cross-linked micelles have been prepared. The micelles exhibited remarkable stability, $\mathrm{pH}$ regulated drug release, good biocompatibility and effective tumor penetration. Cellular uptake demonstrated the efficient endosome/lysosome escape of CFMs, which facilitates the intracellular drug release. Both in vitro and in vivo experiments reflected that CFMs with laser irradiation showed significantly improved therapeutic activity compared with single PDT or chemotherapy. Besides, oxygen-saturated CFMs with laser irradiation exhibited higher cytotoxicity against SKOV3 cells than other formulations, indicating great potential of the synergistic effect. Collectively, CFMs can provide a promising platform for effective diagnosis and treatment of cancer cells, which could substantially improve therapeutic outcomes by chemo-phototherapy.

\section{Ethical Approval}

This study was approved by the Institutional Animal Care and Use Committee of Tianjin Medical University Cancer Institute and Hospital (Permission number: NSFC-AE -2019210). The animals were handled in strict compliance with the Welfare and Ethics of Laboratory Animals Regulations established by the Chinese Association for Laboratory Animal Sciences. 


\section{Funding}

This work was supported by Tianjin Science and Technology Plan (20YFZCSY00040).

\section{Disclosure}

The authors report no conflicts of interest in this work.

\section{References}

1. Liu Y, Bhattarai P, Dai Z, Chen X. Photothermal therapy and photoacoustic imaging: via nanotheranostics in fighting cancer. Chem Soc Rev. 2019;48:2053-2108.

2. Yang N, Xiao W, Song X, Wang W, Dong X. Recent advances in tumor microenvironment hydrogen peroxide-responsive materials for cancer photodynamic therapy. Nano Micro Lett. 2020:1-27.

3. Shi JH, Wang TR, You YQ, et al. Enhancement of ultralow-intensity NIR light-triggered photodynamic therapy based on exo- and endogenous synergistic effects through combined glutathione-depletion chemotherapy. Nanoscale. 2019;11(27):13078-13088. doi:10.1039/ C9NR03052B

4. Wang H, Han RL, Yang LM, et al. Design and synthesis of Core-Shell-Shell upconversion nanoparticles for nir-induced drug release, photodynamic therapy, and cell imaging. ACS Appl Mater Interfaces. 2016;8:4416-4423. doi:10.1021/acsami.5b11197

5. Yuan Y, Min Y, Hu Q, Xing B, Liu B. NIR photoregulated chemoand photodynamic cancer therapy based on conjugated polyelectrolyte-drug conjugate encapsulated upconversion nanoparticles. Nanoscale. 2014;6:11259-11272. doi:10.1039/ C4NR03302G

6. Dang J, He H, Chen D, Yin L. Manipulating tumor hypoxia toward enhanced photodynamic therapy (PDT). Biomater Sci. 2017;5:1500-1511. doi:10.1039/C7BM00392G

7. Phua SZF, Yang G, Lim WQ, et al. Catalase-integrated hyaluronic acid as nanocarriers for enhanced photodynamic therapy in solid tumor. ACS Nano. 2019;13:4742-4751. doi:10.1021/ acsnano.9b01087

8. Fan $\mathrm{W}, \mathrm{Bu} \mathrm{W}$, Shen $\mathrm{B}$, et al. Intelligent $\mathrm{MnO}_{2}$ nanosheets anchored with upconversion nanoprobes for concurrent $\mathrm{pH}-\mathrm{H}_{2} \mathrm{O}_{2}$-responsive UCL imaging and oxygen-elevated synergetic therapy. Adv Mater. 2015;27:4155-4161. doi:10.1002/adma.201405141

9. Lee AL, Gee CT, Weegman BP, et al. Oxygen sensing with perfluorocarbon-loaded ultraporous mesostructured silica nanoparticles. ACS Nano. 2017;11:5623-5632. doi:10.1021/ acsnano.7b01006

10. Hu DR, Zhong L, Wang MY, et al. Perfluorocarbon-loaded and redox-activatable photosensitizing agent with oxygen supply for enhancement of fluorescence/photoacoustic imaging guided tumor photodynamic therapy. Adv Funct Mater. 2019;29:1-14. doi:10.1002/adfm.201806199
11. Xing L, Gong JH, Wang Y, et al. Hypoxia alleviation-triggered enhanced photodynamic therapy in combination with IDO inhibitor for preferable cancer therapy. Biomaterials. 2019;206:170-182. doi:10.1016/j.biomaterials.2019.03.027

12. Pei P, Sun C, Tao W, Li J, Yang X, Wang J. ROS-sensitive thioketal-linked polyphosphoester-doxorubicin conjugate for precise phototriggered locoregional chemotherapy. Biomaterials. 2019;188:74-82. doi:10.1016/j.biomaterials.2018.10.010

13. Li F, Li T, Cao W, Wang L, Xu H. Near-infrared light stimuli-responsive synergistic therapy nanoplatforms based on the coordination of tellurium-containing block polymer and cisplatin for cancer treatment. Biomaterials. 2017;133:208-218. doi:10.1016/j. biomaterials.2017.04.032

14. Gao D, Lo PC. Polymeric micelles encapsulating $\mathrm{pH}$-responsive doxorubicin prodrug and glutathione-activated zinc(II) phthalocyanine for combined chemotherapy and photodynamic therapy. J Control Release. 2018;282:46-61. doi:10.1016/j. jconrel.2018.04.030

15. Lu D, An Y, Feng S, et al. Imidazole-bearing polymeric micelles for enhanced cellular uptake, rapid endosomal escape, and on-demand cargo release. AAPS PharmSciTech. 2018;19:2610-2619. doi:10.1208/s12249-018-1092-2

16. Li X, Gao M, Xin K, et al. Singlet oxygen-responsive micelles for enhanced photodynamic therapy. J Control Release. 2017;260:12-21. doi:10.1016/j.jconrel.2017.05.025

17. Hou G, Qian J, Xu W, et al. Multifunctional PEG-b-polypeptidedecorated gold nanorod for targeted combined chemo-photothermal therapy of breast cancer. Colloids Surf B Biointerfaces. 2019;181:602-611. doi:10.1016/j.colsurfb.2019.05.025

18. Ling X, Chen X, Riddell IA, et al. Glutathione-scavenging Poly (disulfide amide) nanoparticles for the effective delivery of $\mathrm{Pt}(\mathrm{IV})$ prodrugs and reversal of cisplatin resistance. Nano Lett. 2018;18:4618-4625. doi:10.1021/acs.nanolett.8b01924

19. Johnstone TC, Lippard SJ. The effect of ligand lipophilicity on the nanoparticle encapsulation of $\mathrm{Pt}(\mathrm{IV})$ prodrugs. Inorg Chem. 2013;52:9915-9920. doi:10.1021/ic4010642

20. Dhar S, Gu FX, Langer R, Farokhza OC, Lippard SJ. Targeted delivery of cisplatin to prostate cancer cells by aptamer functionalized Pt(IV) prodrug-PLGA-PEG nanoparticles. Proc Natl Acad Sci U S A. 2008;105:17356-17361. doi:10.1073/pnas.0809154105

21. Qiang yuan Z, Liang chen W, Gang You B, et al. Multifunctional nanoparticles co-delivering EZH2 siRNA and etoposide for synergistic therapy of orthotopic non-small-cell lung tumor. $J$ Control Release. 2017;268:198-211. doi:10.1016/j.jconrel.2017.10.025

22. Liu N, Liu H, Chen H, Wang G, Teng H, Chang $Y$. Polyphotosensitizer nanogels for GSH-responsive histone deacetylase inhibitors delivery and enhanced cancer photodynamic therapy. Colloids Surf B Biointerfaces. 2020;188:110753. doi:10.1016/j. colsurfb.2019.110753

\section{Publish your work in this journal}

The International Journal of Nanomedicine is an international, peerreviewed journal focusing on the application of nanotechnology in diagnostics, therapeutics, and drug delivery systems throughout the biomedical field. This journal is indexed on PubMed Central, MedLine, CAS, SciSearch ${ }^{\circledR}$, Current Contents ${ }^{\mathbb{R}} /$ Clinical Medicine,
Journal Citation Reports/Science Edition, EMBase, Scopus and the Elsevier Bibliographic databases. The manuscript management system is completely online and includes a very quick and fair peer-review system, which is all easy to use. Visit http://www.dovepress.com/ testimonials.php to read real quotes from published authors. 